PHYSICAL REVIEW D 91, 024014 (2015)

\title{
Late-time quantum backreaction from inflationary fluctuations of a nonminimally coupled massless scalar
}

\author{
D. Glavan, ${ }^{*}$ T. Prokopec, ${ }^{\dagger}$ and D. C. van der Woude \\ Institute for Theoretical Physics and Spinoza Institute, Center for Extreme Matter \\ and Emergent Phenomena, Science Faculty, Utrecht University, Postbus 80.195, 3508 TD Utrecht, \\ The Netherlands
}

(Received 4 October 2014; published 8 January 2015)

\begin{abstract}
We consider the late-time one-loop quantum backreaction from inflationary fluctuations of a nonminimally coupled, massless scalar field. The scalar is assumed to be a spectator field in an inflationary model with a constant principal slow-roll $\epsilon$ parameter. We regulate the infrared by matching onto a preinflationary radiation era. We find a large late-time backreaction when the nonminimal coupling $\xi$ is negative (in which case the scalar exhibits a negative mass term during inflation). The one-loop quantum backreaction becomes significant today for moderately small nonminimal couplings, $\xi \sim-1 / 20$, and it changes sign (from negative to positive) at a recent epoch when inflation lasts not much longer than what is minimally required, $N \gtrsim 66$. Since currently we do not have a way of treating the classical fluid and the quantum backreaction in a self-consistent manner, we cannot say decidedly whether the backreaction from inflationary quantum fluctuations of a nonminimally coupled scalar can mimic dark energy.
\end{abstract}

DOI: 10.1103/PhysRevD.91.024014

PACS numbers: 04.62.+v, 98.80.-k, 98.80.Qc

\section{INTRODUCTION}

In Ref. [1] we have studied the late-time one-loop quantum backreaction of a minimally coupled scalar field and gravitons from the amplified inflationary vacuum fluctuations on de Sitter space and showed that at late times inflationary fluctuations scale as the (matter) background and contribute only a tiny amount $\left(\sim 10^{-13}\right)$ of the total energy density, thereby constituting a negligible backreaction on the background evolution. This result was subsequently confirmed in [2], where it was also found that the existence of additional pre-inflationary eras and transitions between them can increase the amplitude of the backreaction, but it cannot change its scaling in time. Additionally in [1], by allowing the Hubble parameter to exhibit adiabatic dependence on time, we have made an estimate of the quantum backreaction from scalar cosmological perturbations, and arrived at the conclusion that it is also negligible $\left(\sim 10^{-12}\right)$. The ratio of the tensor and scalar backreactions is approximately equal to the inflationary $r$ parameter, which has recently been measured to be about 0.2 [3]. These considerations refute the claims made some time ago $[4,5]$ that inflationary vacuum fluctuations can play the role of the dark energy today (albeit already soon thereafter that was questioned in Ref. [6]). However, in Ref. [7] it was argued that quantum backreaction from inflationary perturbations of a light, but massive, scalar field can mimic dark energy.

Even though the backreaction from massless minimally coupled scalars and from cosmological perturbations is

\footnotetext{
*D.Glavan@uu.nl

T.Prokopec@uu.nl

†D.C.vanderWoude@uu.nl
}

tiny, it is interesting that it scales precisely as the background, constituting a tiny contribution to dark matter. ${ }^{1}$ This result has inspired us to consider quantum backreaction from inflationary fluctuations of a nonminimally coupled spectator scalar field, because for a particular choice of the nonminimal coupling (which is in our convention negative), the spectrum of amplified vacuum fluctuations can be dramatically red-tilted, thereby enhancing the late-time quantum backreaction, cf. Ref. [8]. At least that was our hope. In this paper we confirm this expectation: for a sufficiently large negative nonminimal coupling $\xi$ latetime backreaction becomes indeed strong. We find that for $\xi<0$ the backreaction grows during inflation, and for a minimal inflation with a number of $e$-folds $N \simeq 66$ (as required by observations), the backreaction is negative and for $\xi \lesssim-0.057$ it exceeds the background density at the end of inflation. This opens the possibility to use quantum fluctuations of a nonminimally coupled scalar to terminate inflation, thus providing a graceful exit from inflation. A proper study of this would require a self-consistent solution of the semiclassical background equation, which is beyond the scope of this paper. At late times during matter era we find that the backreaction is negative for $\xi<0$ and it grows with respect to the background if $\xi<-1 / 3$. However, for

\footnotetext{
${ }^{1}$ It would be of interest to check whether the same can be said about the quantum backreaction from the inflationary perturbations of a light massive scalar field whose mass is smaller than the Hubble rate today $\left(m<H_{0}\right)$, see Ref. [7]. A naive expectation (which would have to be confirmed or rebutted by a detailed analysis) is that the energy density from light inflationary fluctuations scales during inflation as $\rho_{q} \sim H_{I}^{4}$ (where $H_{I}$ is the Hubble rate in inflation), while during radiation and matter eras it scales as $\propto 1 / a^{4}$ and $\propto 1 / a^{3}$,respectively.
} 
these values the backreaction is already too large during inflation, and hence it ought to be discarded. As an interesting case we present a universe with an inflationary period of $N \simeq 69$ and with $\xi \simeq-0.052$. In this case the backreaction is subdominant during inflation and radiation era and it begins negative in matter era, but then it exhibits a transient and becomes positive at recent times, ending up decaying in the future. This case is interesting since it could be potentially used to mimic dark energy. However, a more sophisticated self-consistent analysis of the background evolution that includes the backreaction in the background is needed to properly answer that question, and we postpone this analysis to future work.

The question of quantum backreaction of cosmological perturbations and other quantum fields during inflation is an old subject, and has been extensively studied in literature. A particular attention was devoted to the oneloop quantum backreaction of scalar and tensor cosmological perturbations during inflation [9-16]. A consensus has been reached that a reasonable local observer will observe the local expansion rate [13-15], while a nonlocal observer would observe a backreaction accumulated along the past light cone [9-12]. In both cases the quantum backreaction vanishes at the one-loop order. There are however observers for which the one-loop quantum backreaction does not vanish [17-19], emphasizing the observer's importance when deciding on the quantum backreaction during inflation.

Because of the strongly nonconformal nature of the coupling of scalar and tensor perturbations to an expanding background, these fields are naturally believed to yield a particularly strong quantum backreaction, it is worth investigating the quantum backreaction of other quantum fields, which also (at the classical or quantum level) break conformal invariance. Thus, the two-loop quantum backreaction on de Sitter background was considered in Refs. [20,21], where it was found that the (negative) backreaction (on the background energy density) is of the order, $\sim-10^{-2} \alpha_{e} H^{4} \ln (a)$. On the other hand, its nonperturbative generalization based on stochastic inflation [22] yields at late times in inflation a parametrically larger (negative) backreaction of the order, $\sim-10^{-2} H^{4}$, that is of the order $-G_{N} \Lambda$ times the background density. It is remarkable that this result is independent on the electromagnetic coupling constant $\alpha_{e}$, and hence it is fully nonperturbative. Other results of interest include quantum backreaction in Yukawa theory [23], in which the fermion loops give a negative contribution that grows in magnitude as the scalar field vacuum expectation value, thus destabilizing de Sitter. Finally, it is worth mentioning that the quantum scalar perturbations on de Sitter space are strong enough to restore an $O(N)$ symmetry [24-30], and thus induce a large quantum backreaction [24], albeit it is at the moment not clear whether this effect can have significant late-time impact on the evolution of the Universe and on cosmological perturbations.

\section{FLRW BACKGROUND}

The metric of a $D$-dimensional spatially flat FriedmannLemaître-Robertson-Walker (FLRW) space-time, in conformal time coordinates, is

$$
g_{\mu \nu}(x)=a^{2}(\eta) \eta_{\mu \nu},
$$

where $\eta_{\mu \nu}=\operatorname{diag}(-1,1,1, \ldots, 1)$ is the $D$-dimensional Minkowski metric, and $a(\eta)$ is the scale factor. We work in units $\hbar=c=1$ unless otherwise stated. The conformal time is related to the physical (cosmological) time $t$ as $d t=a(\eta) d \eta$. The conventions we use for the geometric quantities are $\Gamma_{\mu \nu}^{\alpha}=\frac{1}{2} g^{\alpha \beta}\left(\partial_{\mu} g_{\nu \beta}+\partial_{\nu} g_{\mu \beta}-\partial_{\beta} g_{\mu \nu}\right)$ for Christoffel symbols, $R_{\mu \beta \nu}^{\alpha}=\partial_{\beta} \Gamma_{\mu \nu}^{\alpha}-\partial_{\nu} \Gamma_{\mu \beta}^{\alpha}+\Gamma_{\beta \rho}^{\alpha} \Gamma_{\mu \nu}^{\rho}-$ $\Gamma_{\nu \rho}^{\alpha} \Gamma_{\mu \beta}^{\rho}$ for the Riemann tensor, $R_{\mu \nu}=R_{\mu \alpha \nu}^{\alpha}$ for the Ricci tensor, and $R=R_{\mu}^{\mu}$ is the Ricci scalar.

The source for the dynamics of this space-time (the dynamics of the scale factor) is the matter content of the Universe, which we take to be in the form of ideal fluids. Ideal fluids are described by their energy density $\rho$ and pressure $p$. The cosmologically relevant fluids satisfy the linear equation of state $p=w \rho$, where $w_{M}=0$ for nonrelativistic matter (dust), $w_{R}=1 / 3$ for radiation (ultrarelativistic matter), and $w_{\Lambda}=-1$ for cosmological constant.

The dynamics of the FLRW space-time is dictated by the two Friedmann equations,

$$
\begin{gathered}
\left(\frac{\mathcal{H}}{a}\right)^{2}=\frac{8 \pi G_{N}}{3} \sum_{i} \rho_{i}, \\
\frac{\mathcal{H}^{\prime}-\mathcal{H}^{2}}{a^{2}}=-4 \pi G_{N} \sum_{i}\left(\rho_{i}+p_{i}\right),
\end{gathered}
$$

where we have introduced a conformal Hubble rate,

$$
\mathcal{H}=\frac{a^{\prime}}{a},
$$

the prime denoting differentiation with respect to conformal time. It is related to the physical Hubble rate as $H=\mathcal{H} a^{-1}$. Also, $G_{N}$ is the Newton's constant and $c$ the speed of light.

The background ideal fluids satisfy each independently the covariant conservation law,

$$
\rho^{\prime}+3 \mathcal{H}(\rho+p)=0 .
$$

When the equation of state is linear, $p=w \rho$, this conservation law is readily integrated to yield the scaling of energy density and pressure with the scale factor,

$$
\rho=\frac{p}{w}=\rho_{0}\left(\frac{a_{0}}{a}\right)^{3(1+w)},
$$


where $\rho\left(\eta_{0}\right)=\rho_{0}$ and $a\left(\eta_{0}\right)=a_{0}$, and $w$ is the equation of state parameter.

When expansion of the Universe is dominated by a single fluid component (so that other fluids can be neglected), the Friedmann equations can easily be integrated to solve for the scale factor,

$$
a(\eta)=a_{0}\left[1+(\epsilon-1) \mathcal{H}_{0}\left(\eta-\eta_{0}\right)\right]^{\frac{1}{\epsilon-1}},
$$

and the Hubble rate,

$$
\mathcal{H}=\mathcal{H}_{0}\left(\frac{a_{0}}{a}\right)^{\epsilon-1}
$$

where $\mathcal{H}\left(\eta_{0}\right)=\mathcal{H}_{0}$, and $\epsilon$ is the principal slow-roll parameter (the terminology borrowed from inflation, but here it does not have to be small), generally defined as

$$
\epsilon=-\frac{\dot{H}}{H^{2}}=1-\frac{\mathcal{H}^{\prime}}{\mathcal{H}^{2}} .
$$

For spatially flat FLRW space-times dominated by one ideal fluid $\epsilon$ is constant, and depends only on the fluid equation of state parameter,

$$
\epsilon=\frac{3}{2}(1+w) .
$$

The $\epsilon$ parameter is connected to the deceleration parameter as $q=\epsilon-1$, which is usually used to quantify whether and how much the expansion of the Universe is speeding up or slowing down. That is obvious from its definition written in terms of physical time,

$$
q=\epsilon-1=-\frac{\ddot{a} a}{\dot{a}^{2}},
$$

which tells us that the Universe is decelerating when $\epsilon>1$, and it is accelerating when $\epsilon<1$. The relevant values of this parameter are $\epsilon_{M}=3 / 2$ for nonrelativistic matter, $\epsilon_{R}=2$ for radiation and $0<\epsilon_{I} \ll 1$ for inflation (close to that of the cosmological constant $\epsilon_{\Lambda}=0$ ).

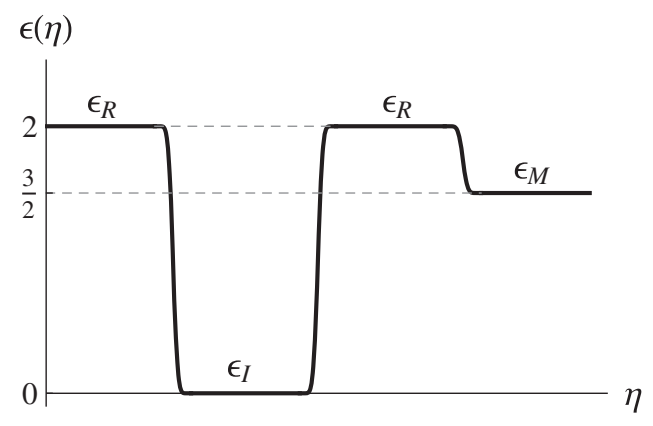

FIG. 1. The evolution of the slow-roll parameter $\epsilon(\eta)$ assumed in this paper.
The picture one should keep in mind when it comes to the expansion history in this paper is shown in Fig. 1. The transition times between periods $\tau_{n}$ are assumed to be fast, $\tau_{n} \ll 1 / \mathcal{H}_{n}$. The initial radiation-dominated period is assumed since it serves as a universal IR regulator [8] for the initial scalar field state.

\section{NONMINIMALLY COUPLED SCALAR FIELD}

The action for a nonminimally coupled massless scalar field is given by

$$
S=\int d^{D} x \sqrt{-g}\left(-\frac{1}{2} g^{\mu \nu} \partial_{\mu} \phi \partial_{\nu} \phi-\frac{1}{2} \xi R \phi^{2}\right),
$$

where the dimensionless parameter $\xi$ is the nonminimal coupling and $g=\operatorname{det}\left(g_{\mu \nu}\right)$. Note that the sign convention for $\xi$ we use is the opposite from the one used in the literature on Higgs inflation, such that $\xi=\xi_{c}=(D-2) /$ $[4(D-1)]$ is the conformal coupling in our convention. In order to quantize this field on a FLRW background we first define a canonically conjugate momentum

$$
\pi(x)=\frac{\delta S}{\delta \phi^{\prime}(x)}=a^{D-2}(\eta) \phi^{\prime}(x),
$$

then promote $\phi$ and $\pi$ to operators and impose canonical commutation relations (we work in the Heisenberg picture),

$$
\begin{aligned}
{\left[\hat{\phi}(\eta, \boldsymbol{x}), \hat{\pi}\left(\eta, \boldsymbol{x}^{\prime}\right)\right] } & =i \delta^{D-1}\left(\boldsymbol{x}-\boldsymbol{x}^{\prime}\right), \\
{\left[\hat{\phi}(\eta, \boldsymbol{x}), \hat{\phi}\left(\eta, \boldsymbol{x}^{\prime}\right)\right] } & =0=\left[\hat{\pi}(\eta, \boldsymbol{x}), \hat{\pi}\left(\eta, \boldsymbol{x}^{\prime}\right)\right] .
\end{aligned}
$$

The Heisenberg equations then give us the equation of motion for the field operator,

$$
\begin{aligned}
\hat{\phi}^{\prime \prime} & +(D-2) \mathcal{H} \hat{\phi}^{\prime}-\nabla^{2} \hat{\phi}+\xi(D-1)\left(2 \mathcal{H}^{\prime}+(D-2) \mathcal{H}^{2}\right) \hat{\phi} \\
& =0,
\end{aligned}
$$

and we have assumed that gravity is nondynamical (for a discussion of the one-loop graviton contribution see Ref. [1]). Due to the spatial translation and rotation invariance of the background, it is convenient to decompose the field operator into Fourier modes,

$$
\begin{aligned}
\hat{\phi}(\eta, \boldsymbol{x})= & a^{\frac{2-D}{2}} \int \frac{d^{D-1} k}{(2 \pi)^{D-1}}\left(e^{i \boldsymbol{k} \cdot \boldsymbol{x}} U(k, \eta) \hat{b}(\boldsymbol{k})\right. \\
& \left.+e^{-i \boldsymbol{k} \cdot \boldsymbol{x}} U^{*}(k, \eta) \hat{b}^{\dagger}(\boldsymbol{k})\right),
\end{aligned}
$$

where the mode function $U(k, \eta)$ is assumed to depend only on the modulus of the momentum $k=\|\boldsymbol{k}\|$ (corresponding to an isotropic state). Its equation of motion is

$$
U^{\prime \prime}(k, \eta)+\left(k^{2}+f(\eta)\right) U(k, \eta)=0,
$$


where

$$
f(\eta)=-\frac{1}{4}(D-2-4 \xi(D-1))\left(2 \mathcal{H}^{\prime}+(D-2) \mathcal{H}^{2}\right) .
$$

We require the annihilation and creation operators to satisfy the following commutation relations:

$$
\begin{gathered}
{\left[\hat{b}(\boldsymbol{k}), \hat{b}^{\dagger}\left(\boldsymbol{k}^{\prime}\right)\right]=(2 \pi)^{D-1} \delta^{D-1}\left(\boldsymbol{k}-\boldsymbol{k}^{\prime}\right),} \\
{\left[\hat{b}(\boldsymbol{k}), \hat{b}\left(\boldsymbol{k}^{\prime}\right)\right]=0=\left[\hat{b}^{\dagger}(\boldsymbol{k}), \hat{b}^{\dagger}\left(\boldsymbol{k}^{\prime}\right)\right],}
\end{gathered}
$$

which then fixes the Wronskian normalization of the mode function

$$
\begin{aligned}
\mathcal{W} & {\left[U(k, \eta), U^{*}(k, \eta)\right] } \\
& =U(k, \eta) \frac{\partial}{\partial \eta} U^{*}(k, \eta)-U^{*}(k, \eta) \frac{\partial}{\partial \eta} U(k, \eta)=i .
\end{aligned}
$$

The vacuum state $|\Omega\rangle$ is defined to be annihilated by the annihilation operators,

$$
\hat{b}(\boldsymbol{k})|\Omega\rangle=0, \quad \forall \boldsymbol{k},
$$

and the entire Fock space is constructed by acting on it by the creation operators $\hat{b}^{\dagger}(\boldsymbol{k})$. It is completely determined once we have specified what is the mode function and its derivative at some time. The initial state we pick is the Bunch-Davies (BD) vacuum $u(k, \eta)$, which is the one that reduces to the flat space positive-frequency mode function in the UV,

$$
U(k, \eta) \stackrel{\mathrm{BD}}{\longrightarrow} u(k, \eta) \stackrel{k \rightarrow \infty}{\sim} \frac{e^{-i k \eta}}{\sqrt{2 k}}
$$

since the high momentum modes are insensitive to curvature, as can be seen e.g. from the UV adiabatic expansion. Physically, this simply means that exciting high momentum modes costs a lot of energy, and is therefore highly suppressed. On constant $\epsilon$ backgrounds (with which we will be concerned here) it is possible to extend definition (22) to a global Bunch-Davies state, which will be given explicitly in Sec. V (for a more detailed discussion on this see Ref. [1]).

\section{ENERGY-MOMENTUM TENSOR}

The energy-momentum tensor operator of a massless nonminimally coupled scalar field is defined to be

$$
\begin{aligned}
\hat{T}_{\mu \nu}= & \left.\frac{-2}{\sqrt{-g}} \frac{\delta S}{\delta g^{\mu \nu}}\right|_{\phi \rightarrow \hat{\phi}} \\
= & \partial_{\mu} \hat{\phi} \partial_{\nu} \hat{\phi}-\frac{1}{2} g_{\mu \nu} g^{\alpha \beta} \partial_{\alpha} \hat{\phi} \partial_{\beta} \hat{\phi} \\
& +\xi\left(G_{\mu \nu}-\nabla_{\mu} \nabla_{\nu}+g_{\mu \nu} \square\right) \hat{\phi}^{2},
\end{aligned}
$$

where $G_{\mu \nu}=R_{\mu \nu}-\frac{1}{2} g_{\mu \nu} R$ is the Einstein tensor, $\nabla$ denotes the covariant derivative, and $\square=g^{\mu \nu} \nabla_{\mu} \nabla_{\nu}$. The expectation value of this operator on FLRW with respect to the isotropic state $|\Omega\rangle$ (defined in the previous section) is diagonal, and it can be written as integrals in Fourier space over squares of the mode function,

$$
\begin{aligned}
\left\langle\Omega\left|\hat{T}_{00}\right| \Omega\right\rangle= & \frac{a^{2-D}}{(4 \pi)^{\frac{D-1}{2}} \Gamma\left(\frac{D-1}{2}\right)} \int_{0}^{\infty} d k k^{D-2} \\
& \times\left[2 k^{2}|U|^{2}-\frac{1}{2}(D-2-4 \xi(D-1)) \mathcal{H}^{\prime}|U|^{2}\right. \\
& \left.-\frac{1}{2}(D-2-4 \xi(D-1)) \mathcal{H} \frac{\partial}{\partial \eta}|U|^{2}+\frac{1}{2} \frac{\partial^{2}}{\partial \eta^{2}}|U|^{2}\right],
\end{aligned}
$$

$$
\begin{aligned}
\left\langle\Omega\left|\hat{T}_{i j}\right| \Omega\right\rangle= & \frac{\delta_{i j} a^{2-D}}{(4 \pi)^{\frac{D-1}{2}} \Gamma\left(\frac{D-1}{2}\right)} \int_{0}^{\infty} d k k^{D-2} \\
& \times\left[\frac{2 k^{2}}{D-1}|U|^{2}-\frac{1}{2}(D-2-4 \xi(D-1)) \mathcal{H}^{\prime}|U|^{2}\right. \\
& -\frac{1}{2}(D-2-4 \xi(D-1)) \mathcal{H} \frac{\partial}{\partial \eta}|U|^{2} \\
& \left.+\frac{1}{2}(1-4 \xi) \frac{\partial^{2}}{\partial \eta^{2}}|U|^{2}\right],
\end{aligned}
$$

where we have exploited the fact that the mode function depends only on the modulus of the momentum and performed the angular integrations right away. In addition, we have used the equation of motion (17) to rewrite $\left|U^{\prime}\right|^{2}=\left(k^{2}+f\right)|U|^{2}+\frac{1}{2} \frac{\partial^{2}}{\partial \eta^{2}}|U|^{2}$. Note that since $\langle\Omega|\hat{\phi}(x)| \Omega\rangle=0$ for all times, the expressions above represent a purely quantum contribution to the energymomentum tensor.

The two quantities above are divergent in $D=4$ (quartically, quadratically and logarithmically); hence they need to be regularized and renormalized. The regularization used here is the dimensional regularization (that is why we kept all the expressions in arbitrary $D$ dimensions) which automatically removes the quartic and quadratic divergences. As shown in Appendix A, the logarithmic divergences are absorbed into higher-derivative counterterms, after which the limit $D \rightarrow 4$ can be taken and finite physical values associated with the two integrals above. From now on we express this expectation value of the energymomentum tensor in terms of the energy density and pressure (of the quantum fluid),

$$
\rho_{q}=\frac{1}{a^{2}}\left\langle\Omega\left|\hat{T}_{00}\right| \Omega\right\rangle, \quad \delta_{i j} p_{q}=\frac{1}{a^{2}}\left\langle\Omega\left|\hat{T}_{i j}\right| \Omega\right\rangle .
$$

The ratio of these two quantities is the equation of state parameter for the quantum fluid, 


$$
w_{q}=\frac{p_{q}}{\rho_{q}},
$$

which is sometimes a convenient quantity to use when comparing it to the classical fluid which drives the expansion. This energy density and pressure have to satisfy the conservation equation

$$
\rho_{q}^{\prime}+3 \mathcal{H}\left(\rho_{q}+p_{q}\right)=0
$$

which also serves as an independent check of the calculation.

\section{EVOLUTION OF THE MODE FUNCTION AND BOGOLYUBOV COEFFICIENTS}

Since we will be concerned with the scalar field evolving on FLRW with a few periods of constant $\epsilon$ expansion (as shown in Fig. 1), it is convenient to examine what the Bunch-Davies state is on those periods. On accelerating periods $(\epsilon<1)$ the BD mode function is [8]

$$
u(k, \eta)=\sqrt{\frac{\pi}{4(1-\epsilon) \mathcal{H}}} H_{\nu}^{(1)}\left(\frac{k}{(1-\epsilon) \mathcal{H}}\right),
$$

and on decelerating periods $(\epsilon>1)$ we take it to be

$$
u(k, \eta)=\sqrt{\frac{\pi}{4(\epsilon-1) \mathcal{H}}} H_{\nu}^{(2)}\left(\frac{k}{(\epsilon-1) \mathcal{H}}\right),
$$

where in both cases

$$
\nu^{2}=\frac{1}{4}+\frac{D-2 \epsilon}{4(1-\epsilon)^{2}}(D-2-4 \xi(D-1)),
$$

and $H_{\nu}^{(1)}$ and $H_{\nu}^{(2)}$ are the first and second Hankel functions, respectively. ${ }^{2}$

Even if the field starts in a BD state during some period of constant $\epsilon=\epsilon_{0}$, if $\epsilon$ evolves, so will the state and when $\epsilon$ eventually settles to a new constant value $\epsilon=\epsilon_{1} \neq \epsilon_{0}$, the state will differ from the BD state with $\epsilon=\epsilon_{1}$ [characterized by $u(k, \eta)]$. The scalar mode function can then be written as

$$
U(k, \eta)=\alpha(k) u(k, \eta)+\beta(k) u^{*}(k, \eta),
$$

\footnotetext{
${ }^{2}$ Our choice for the BD mode function on decelerating periods might seem odd, since usually (29) is a BD mode function on any constant $\epsilon$ period. But, since we assume that $\nu$ is real (in order to have appreciable particle production during inflation), $H_{\nu}^{(1)}(-x)$ differs from $H_{\nu}^{(2)}(x)$ by an irrelevant phase factor, our choice corresponds to picking a particular phase which does not affect physical quantities. This way, the arguments of the Hankel functions are always positive, which we find more convenient to work with.
}

where $\alpha$ and $\beta$ are the Bogolyubov coefficients which, because of the space-time homogeneity, depend on the modulus of the momentum, and do not mix different modes since the equation of the motion for the field is linear. This is nothing other than representing the full solution to the equation of motion (17) in the basis of BD solutions. The actual form of the Bogolyubov coefficients depends on the exact evolution of the background from one period to the other. The canonical quantization puts the following constraint on these coefficients:

$$
|\alpha(k)|^{2}-|\beta(k)|^{2}=1,
$$

which is dictated by the Wronskian normalization (20). The evolution of the mode function on a smooth FLRW background also puts constraints on these coefficients in the UV. Namely, they have to reduce to $\alpha \rightarrow 1$ and $\beta \rightarrow 0$ as $k \rightarrow \infty$ faster than any power of $1 / k$ (see Appendix A).

Before dealing with the specific structure of the Bogolyubov coefficients, we will first define a convenient way to split the expectation value for the energy-momentum tensor. On a given $n$-th period of constant $\epsilon_{n}$ the mode function will be given as

$$
U_{n}(k, \eta)=\alpha_{n}(k) u_{n}(k, \eta)+\beta_{n}(k) u_{n}^{*}(k, \eta),
$$

where $u_{n}(k, \eta)$ is the Bunch-Davies mode function on a given $n$th period. Since in the integrals (24) and (25) only $\left|U_{n}\right|^{2}$ is needed, we find it convenient to write it out in terms of BD mode function as

$$
\left|U_{n}\right|^{2}=\left|u_{n}\right|^{2}+\left[2\left|\beta_{n}\right|^{2}\left|u_{n}\right|^{2}+\alpha_{n} \beta_{n}^{*} u_{n}^{2}+\alpha_{n}^{*} \beta_{n} u_{n}^{* 2}\right],
$$

where (33) was used. To facilitate our analysis, it is convenient to define the energy density and pressure functionals,

$$
\begin{aligned}
\tilde{\rho}_{q}[Z]= & \frac{a^{-D}}{(4 \pi)^{\frac{D-1}{2}} \Gamma\left(\frac{D-1}{2}\right)} \int_{k_{0}}^{\infty} d k k^{D-2}\left[2 k^{2} Z(k, \eta)\right. \\
& +\frac{1}{2}(D-2-4 \xi(D-1))\left(\epsilon_{n}-1\right) \mathcal{H}^{2} Z(k, \eta) \\
& -\frac{1}{2}(D-2-4 \xi(D-1)) \mathcal{H} \frac{\partial}{\partial \eta} Z(k, \eta) \\
& \left.+\frac{1}{2} \frac{\partial^{2}}{\partial \eta^{2}} Z(k, \eta)\right], \\
\tilde{p}_{q}[Z]= & \frac{a^{-D}}{(4 \pi)^{\frac{D-1}{2}} \Gamma\left(\frac{D-1}{2}\right)} \int_{k_{0}}^{\infty} d k k^{D-2}\left[\frac{2 k^{2}}{D-1} Z(k, \eta)\right. \\
& +\frac{1}{2}(D-2-4 \xi(D-1))\left(\epsilon_{n}-1\right) \mathcal{H}^{2} Z(k, \eta) \\
& -\frac{1}{2}(D-2-4 \xi(D-1)) \mathcal{H} \frac{\partial}{\partial \eta} Z(k, \eta) \\
& \left.+\frac{1}{2}(1-4 \xi) \frac{\partial^{2}}{\partial \eta^{2}} Z(k, \eta)\right],
\end{aligned}
$$


where then the full energy density and pressure are sums of two contributions, one with $Z=Z_{B D}$ and another with $Z=Z_{\text {Bog }}$. (to be defined below). The IR regulator $k_{0}$ introduced here does not mean the state is IR divergent and needs regularization (starting with an initial radiation period takes care of that [31]). It just means that the individual integrals in which we have split the expectation value might be IR divergent individually. In the final answer, when they are all added together, the IR divergent terms cancel and the limit $k_{0} \rightarrow 0$ can safely be taken.

The first part of the energy density and pressure (24)-(26) corresponds to the contribution one would get from assuming a BD vacuum state on a given period (so in that sense this part sees no transitions),

$$
\rho_{q}^{B D}=\tilde{\rho}_{q}\left[\left|u_{n}\right|^{2}\right], \quad p_{q}^{B D}=\tilde{p}_{q}\left[\left|u_{n}\right|^{2}\right] .
$$

This BD part contains all UV divergences of the energymomentum tensor, and the regularization and renormalization procedure outlined in Appendix A applies to this contribution. The remaining contributions will not contain any UV divergences. Since all possible $k_{0}$-dependent terms cancel, on dimensional grounds, the contribution of the BD part must be proportional to $\mathcal{H}^{4}$. The rest of the time dependence is $a^{-4} \ln (a)$ at best (Appendix A). On a matterdominated period $\mathcal{H}$ falls off as $a^{-1 / 2}$, meaning this BD contribution to the energy density and pressure is negligible at late times compared to the background quantities, and we will not consider it in the following.

The remaining contributions to the energy density and pressure involve integrals over Bogolyubov coefficients as well, involving in particular $\beta(k)$, which is nonadiabatically (faster than any power law) suppressed in the UV, meaning that the integrals can be evaluated in $D=4$ right away. These contributions are

$\rho_{q}^{\mathrm{Bog}}=\tilde{\rho}_{q}\left[Z_{\mathrm{Bog}}(k, \eta)\right], \quad p_{q}^{\mathrm{Bog}}=\tilde{p}_{q}\left[Z_{\mathrm{Bog}}(k, \eta)\right]$,

where

$$
\begin{aligned}
Z_{\mathrm{Bog}}(k, \eta)= & 2\left|\beta_{n}(k)\right|^{2}\left|u_{n}(k, \eta)\right|^{2}+\alpha_{n}(k) \beta_{n}^{*}(k) u_{n}^{2}(k, \eta) \\
& +\alpha_{n}^{*}(k) \beta_{n}(k) u_{n}^{* 2}(k, \eta) .
\end{aligned}
$$

If there are any interesting effects that depend on the expansion history of the Universe, they must lie in these contributions, since $\alpha_{n}$ and $\beta_{n}$ carry all the information about the expansion. Therefore, we devote the rest of the paper to calculating this part.

Calculating (39) can be reduced to calculating integrals,

$$
\mathcal{I}_{s}=\int_{k_{0}}^{\infty} d k k^{2 s} Z_{\mathrm{Bog}}(k, \eta), \quad s=1,2,
$$

and then just acting on them with differential operators,

$$
\begin{aligned}
\rho_{q} \approx \rho_{q}^{\mathrm{Bog}}= & \frac{1}{4 \pi^{2} a^{4}}\left\{2 \mathcal{I}_{2}+\left[(1-6 \xi)\left(\epsilon_{n}-1\right) \mathcal{H}^{2}\right.\right. \\
& \left.\left.-(1-6 \xi) \mathcal{H} \frac{\partial}{\partial \eta}+\frac{1}{2} \frac{\partial^{2}}{\partial \eta^{2}}\right] \mathcal{I}_{1}\right\}, \\
p_{q} \approx p_{q}^{\text {Bog }}= & \frac{1}{4 \pi^{2} a^{4}}\left\{\frac{2}{3} \mathcal{I}_{2}+\left[(1-6 \xi)\left(\epsilon_{n}-1\right) \mathcal{H}^{2}\right.\right. \\
& \left.\left.-(1-6 \xi) \mathcal{H} \frac{\partial}{\partial \eta}+\frac{1}{2}(1-4 \xi) \frac{\partial^{2}}{\partial \eta^{2}}\right] \mathcal{I}_{1}\right\} .
\end{aligned}
$$

Isolating the dominant contributions to the integrands in integrals (41) and calculating the integrals comprises the main part of this work.

\section{DOMINANT CONTRIBUTIONS TO INTEGRALS $\mathcal{I}_{s}$}

In this section we argue where dominant contributions to integrals (41) should lie. We do this by approximating the integrand function $Z_{\mathrm{Bog}}(k, \eta)$ on different integration intervals in such a way that the integration can be performed. Approximations in essence consist of performing expansions in certain small ratios of scales.

The transitions between the periods are assumed fast, so the Hubble rate at the time of transition is a well-defined quantity, since it varies very little during the time of the transition $\tau \ll 1 / \mathcal{H}$. Schematically, the evolution of the Hubble rate is depicted in Fig. 2.

In particular, the hierarchy we are dealing with is $\mathcal{H}, \mathcal{H}_{0} \ll \mathcal{H}_{2} \ll \mathcal{H}_{1} \ll \tau^{-1}$, where $\tau$ is some scale of transition. Therefore, we first split the integration into the IR and UV part with respect to scales $\mathcal{H}, \mathcal{H}_{0}$. We do this by introducing a comoving cutoff $\mu$ such that $\mathcal{H}_{0}, \mathcal{H} \ll \mu \ll \mathcal{H}_{2} \ll \mathcal{H}_{1}$.

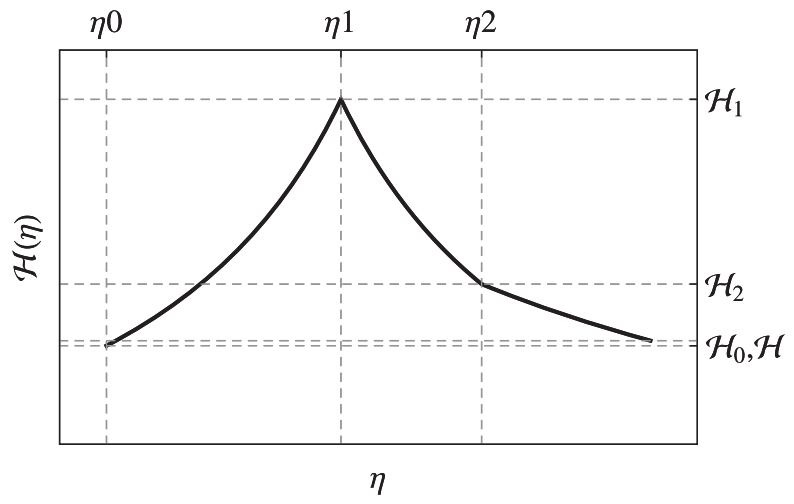

FIG. 2. Schematic evolution of the conformal Hubble rate during different expansion periods. The beginning of inflation is denoted by $\eta_{0}, \eta_{1}$ denotes the start of radiation period, and $\eta_{2}$ the start of matter period. The hierarchy between scales today is $\mathcal{H}_{1} \gg \mathcal{H}_{2} \gg \mathcal{H} \sim \mathcal{H}_{0}$. 


\section{A. UV contribution}

First we want to argue that the relevant contribution (if it exists) comes from the IR part of the integral $(0, \mu)$. Consider the UV integral

$$
\begin{aligned}
I_{s}^{(\mu, \infty)} & =\int_{\mu}^{\infty} d k k^{2 s} Z_{\mathrm{Bog}}(k, \eta) \\
& =\int_{\mu}^{\infty} d k k^{2 s}\left[2\left|\beta_{M}\right|^{2}\left|u_{M}\right|^{2}+\alpha_{M} \beta_{M}^{*} u_{M}^{2}+\alpha_{M}^{*} \beta_{M} u_{M}^{* 2}\right] .
\end{aligned}
$$

The dependence on the momentum in functions $u_{M}$ comes as $k / \mathcal{H}$. Since $\mu \gg \mathcal{H}$ the argument of this function is always very big, and we can expand these mode functions asymptotically (see Appendix A),

$$
\begin{aligned}
& \left|u_{M}\right|^{2}=\frac{1}{2 k}\left[1+\mathcal{O}\left(\mathcal{H}^{2}\right)\right], \\
& u_{M}^{2}=\frac{1}{2 k} e^{-\frac{2 i k}{\mathcal{H}}}[1+\mathcal{O}(\mathcal{H})],
\end{aligned}
$$

and we have for the integral

$$
\begin{aligned}
I_{s}^{(\mu, \infty)} \approx & \frac{1}{2} \int_{\mu}^{\infty} d k k^{2 s-1}\left[2\left|\beta_{M}\right|^{2}+\alpha_{M} \beta_{M}^{*} e^{-\frac{4 i k}{\mathcal{H}}}+\alpha_{M}^{*} \beta_{M} e^{\frac{4 i k}{\mathcal{H}}}\right] \\
& \leq \frac{1}{2} \int_{\mu}^{\infty} d k k^{2 s-1} 2\left|\beta_{M}\right|^{2}+\left|\frac{1}{2} \int_{\mu}^{\infty} d k k^{2 s-1} \alpha_{M} \beta_{M}^{*} e^{-\frac{4 i k}{\mathcal{H}}}\right| \\
& +\left|\frac{1}{2} \int_{\mu}^{\infty} d k k^{2 s-1} \alpha_{M}^{*} \beta_{M} e^{\frac{4 i k}{\mathcal{H}}}\right| \\
& \leq \frac{1}{2} \int_{\mu}^{\infty} d k k^{2 s-1}\left[2\left|\beta_{M}\right|^{2}+2\left|\alpha_{M} \beta_{M}^{*}\right|\right] .
\end{aligned}
$$

The dominant contribution to this integral is a constant (does not evolve in time), which means that the dominant contribution to energy density and pressure scales like radiation. Since $\beta$ Bogolyubov coefficient is at least exponentially suppressed in the UV, $\beta \sim e^{-\tau k}$, this contribution has to be small, $\sim \mu^{2 s} e^{-\tau \mu}$, and since it scales like radiation it falls off with time with respect to the background becoming more and more negligible. Non-negligible contributions, if they exist come from the IR effects and should be isolated from the IR part of $\mathcal{I}_{s}$ integral.

\section{B. IR contribution}

The IR contribution to $\mathcal{I}_{s}$ integrals,

$$
\mathcal{I}_{s}^{(0, \mu)}=\int_{k_{0}}^{\mu} d k k^{2 s} Z_{\mathrm{Bog}}(k, \eta),
$$

is more difficult to evaluate, since the IR modes evolve nonadiabatically and are sensitive to the expansion history of the Universe (Fig. 1). The main problem is determining the Bogolyubov coefficients generated by the transitions between different cosmological eras.

For fast (monotonous) transitions between different periods of constant $\epsilon$ the IR modes oscillate slowly enough not to be sensitive to the details of the transitions. Therefore, we expect them to be well described by the so-called sudden transition approximation, where the background $\epsilon$ parameter suddenly jumps from one value to another. This picture should not be taken too literally as the background model of the expansion of the Universe, rather as an expansion in powers of the small time of transition. Also, this approximation, although being very good in the IR, fails in the UV, where it spoils adiabaticity of the UV modes leading to additional unphysical UV divergences of the energy-momentum tensor (see [1] for a detailed discussion), and it should not be used there. Once again, even though in what follows we treat the background as being one with sudden transitions, we emphasize that this is an approximation for the evolution of the IR modes, not an approximation for the background itself.

\section{SUDDEN TRANSITION APPROXIMATION}

Let us consider a sudden transition from a period of constant $\epsilon_{0}$ to a period of constant $\epsilon_{1}$ at some time $\eta=\eta_{0}$. If the scalar mode function before the transition was $U_{0}(k, \eta)$, then the mode function after the transition will be

$$
U_{1}(k, \eta)=\alpha_{1,0}(k) u_{1}(k, \eta)+\beta_{1,0}(k) u_{1}^{*}(k, \eta),
$$

where $u_{1}$ is the BD mode function for a constant $\epsilon=\epsilon_{1}$ of the later period, and $\alpha_{1,0}(k), \beta_{1,0}(k)$ are the Bogolyubov coefficients determined by the continuity conditions on the mode function and its derivative at the transition time,

$\alpha_{1,0}(k)=i\left[U_{0}^{\prime}\left(k, \eta_{0}\right) u_{1}^{*}\left(k, \eta_{0}\right)-U_{0}\left(k, \eta_{0}\right) u_{1}^{\prime *}\left(k, \eta_{0}\right)\right]$,

$\beta_{1,0}(k)=i\left[U_{0}\left(k, \eta_{0}\right) u_{1}^{\prime}\left(k, \eta_{0}\right)-U_{0}^{\prime}\left(k, \eta_{0}\right) u_{1}\left(k, \eta_{0}\right)\right]$.

If we are dealing with a sequence of sudden transitions, starting with a BD state during the period $\epsilon=\epsilon_{0}$, it is easy to see that the final Bogolyubov coefficients during period $\epsilon_{n}$ will be

$$
\begin{aligned}
\left(\begin{array}{c}
\alpha_{n, 0}(k) \\
\beta_{n, 0}(k)
\end{array}\right)= & \boldsymbol{T}_{n}\left(k, \eta_{n-1}\right) \boldsymbol{T}_{n-1}\left(k, \eta_{n-2}\right) \ldots \boldsymbol{T}_{2}\left(k, \eta_{1}\right) \\
& \times \boldsymbol{T}_{1}\left(k, \eta_{0}\right)\left(\begin{array}{l}
1 \\
0
\end{array}\right)
\end{aligned}
$$

where the transfer matrices are

$$
\boldsymbol{T}_{i}\left(k, \eta_{i-1}\right)=\left(\begin{array}{ll}
\alpha_{i, i-1}\left(k, \eta_{i-1}\right) & \beta_{i, i-1}^{*}\left(k, \eta_{i-1}\right) \\
\beta_{i, i-1}\left(k, \eta_{i-1}\right) & \alpha_{i, i-1}^{*}\left(k, \eta_{i-1}\right)
\end{array}\right),
$$

and the partial Bogolyubov coefficients are defined to be 


$$
\begin{aligned}
\alpha_{i, i-1}\left(k, \eta_{i-1}\right)= & i\left[u_{i}^{*}\left(k, \eta_{i-1}\right) u_{i-1}^{\prime}\left(k, \eta_{i-1}\right)\right. \\
& \left.-u_{i}^{\prime *}\left(k, \eta_{i-1}\right) u_{i-1}\left(k, \eta_{i-1}\right)\right], \\
\beta_{i, i-1}\left(k, \eta_{i-1}\right)= & i\left[u_{i}^{\prime}\left(k, \eta_{i-1}\right) u_{i-1}\left(k, \eta_{i-1}\right)\right. \\
& \left.-u_{i}\left(k, \eta_{i-1}\right) u_{i-1}^{\prime}\left(k, \eta_{i-1}\right)\right],
\end{aligned}
$$

and are composed only of BD mode functions and their derivatives evaluated at the transition time.

\section{A. Bogolyubov coefficients in the UV}

These Bogolyubov coefficients (52) should capture the time evolution of the IR modes (the definition of which somewhat changes during evolution). But the UV modes undergo a different type of evolution, namely adiabatic. Since it costs a lot of energy to excite the UV modes, we expect them not to have a significant contribution to the energy density and pressure in the end. Therefore, the exact structure of the Bogolyubov coefficients in the UV should not be relevant, as long as the unitarity condition (33) is satisfied, and $|\alpha| \rightarrow 1, \beta \rightarrow 0$ faster than any power of $1 / k$ as $k \rightarrow \infty$. A simple way to model this is to construct the full Bogolyubov coefficients out of the sudden transition ones in the following manner:

$$
\begin{aligned}
& \beta_{i, i-1} \rightarrow \beta_{i, i-1} e^{-k \tau_{i-1}}, \\
& \alpha_{i, i-1} \rightarrow \sqrt{1+\beta_{i, i-1} e^{-k \tau_{i-1}}} \frac{\alpha_{i, i-1}}{\left|\alpha_{i, i-1}\right|} .
\end{aligned}
$$

They quickly reduce to the sudden transition ones in the IR, and are nonadiabatically suppressed in the UV, and satisfy (33) by construction. The point of this regularization is not to represent exactly the evolution of the mode function, but rather it allows us to extract the leading order behavior of the energy-momentum tensor while making intermediate steps of the calculation well defined. Parameters $\tau_{i}$ have clear physical interpretation as the durations of transitions, and are (in principle) an independent scale of the system. By introducing them we have control over the approximation for fast transitions, and the expansion in $\tau_{i}$ 's yields the leading behavior. ${ }^{3}$ Calculating corrections of order $\tau_{i}$ and higher to the result does not make much sense.

\footnotetext{
${ }^{3}$ In [2] the sudden transition Bogolyubov coefficients were regulated by cutting them off sharply at the Hubble rate $\mathcal{H}$ of the transition, $\alpha(k)=1, \beta(k)=0$ for $k>\mathcal{H}$. This way the durations of transitions are no longer independent scales, but are intimately tied with the Hubble rate of the transition, and they are not short compared to the Hubble rate either. Such approach accounts for all the terms in the energy density and pressure when it comes to their scaling in time (see Appendix D in [1]), but their coefficients (in principle) cannot be calculated reliably because the two scales describing the transition were assumed the same from the start, and there is no control over the expansion in the duration of transitions. Though the leading order results in [1] and [2] agree.
}

\section{B. Bogolyubov coefficients in the deep IR}

In this subsection we present the IR expansion of the Bogolyubov coefficients generated by $n$ transitions between periods of constant $\epsilon$. This was already derived, in a much more general setting of smooth transitions in [32]. Here we present the expansion for the special case of sudden transitions in a form suited for our needs.

Consider a sudden transition between two decelerating periods $\epsilon_{0}$ and $\epsilon_{1}$. It is convenient to write the $\mathrm{BD}$ mode function (30) as a power series around $k=0$ (which is just the definition of the Bessel function),

$$
\begin{aligned}
u(k, \eta)= & \sqrt{\frac{\pi}{4(\epsilon-1) \mathcal{H}}} \frac{i}{\sin (\pi \nu)}\left[J_{-\nu}\left(\frac{k}{(\epsilon-1) \mathcal{H}}\right)\right. \\
& \left.-e^{i \pi \nu} J_{\nu}\left(\frac{k}{(\epsilon-1) \mathcal{H}}\right)\right] \\
= & \sqrt{\frac{\pi}{4(\epsilon-1) \mathcal{H}}} \frac{i}{\sin (\pi \nu)}\left[\frac{[2(\epsilon-1) \mathcal{H}]^{\nu}}{k^{\nu}} S_{-\nu}\left(\frac{k}{(\epsilon-1) \mathcal{H}}\right)\right. \\
& \left.-e^{i \pi \nu} \frac{k^{\nu}}{[2(\epsilon-1) \mathcal{H}]^{\nu}} S_{\nu}\left(\frac{k}{(\epsilon-1) \mathcal{H}}\right)\right],
\end{aligned}
$$

and similarly for the case of a $\epsilon<1$ BD mode function, where

$$
S_{ \pm \nu}(z)=\sum_{l=0}^{\infty} \frac{(-1)^{l}}{l ! \Gamma(l \pm \nu+1)}\left(\frac{z}{2}\right)^{2 l}=\frac{1}{\Gamma(1 \pm \nu)}+\mathcal{O}\left(z^{2}\right) .
$$

We will be interested in the leading order term in the Bogolyubov coefficients, so for noninteger $\nu$ it suffices to keep only the first term in the series for $S_{ \pm \nu}(z) \simeq 1 / \Gamma(1 \pm \nu)$ from the start. Therefore, it is enough to work with

$$
u_{n}(k, \eta)=i M_{n}(\mathcal{H}) k^{-\nu_{n}}-i e^{i \pi \nu_{n}} P_{n}(\mathcal{H}) k^{\nu_{n}}
$$

for $\epsilon_{n}>1$, and with

$$
u_{n}(k, \eta)=-i M_{n}(\mathcal{H}) k^{-\nu_{n}}+i e^{-i \pi \nu_{n}} P_{n}\left(\mathcal{H}_{n}\right) k^{\nu_{n}}
$$

for $\epsilon_{n}<1$, where

$$
\begin{aligned}
M_{n}(\mathcal{H}) & =\sqrt{\frac{\pi}{2}} \frac{\left[2\left|\epsilon_{n}-1\right| \mathcal{H}\right]^{\nu_{n}-\frac{1}{2}}}{\sin \left(\pi \nu_{n}\right) \Gamma\left(1-\nu_{n}\right)} \\
& =\frac{\Gamma\left(\nu_{n}\right)}{\sqrt{2 \pi}}\left[2\left|\epsilon_{n}-1\right| \mathcal{H}\right]^{\nu_{n}-\frac{1}{2}}, \\
P_{n}(\mathcal{H}) & =\sqrt{\frac{\pi}{2}} \frac{\left[2\left|\epsilon_{n}-1\right| \mathcal{H}\right]^{-\nu_{n}-\frac{1}{2}}}{\sin \left(\pi \nu_{n}\right) \Gamma\left(1+\nu_{n}\right)} \\
& =-\frac{\Gamma\left(-\nu_{n}\right)}{\sqrt{2 \pi}}\left[2\left|\epsilon_{n}-1\right| \mathcal{H}\right]^{-\nu_{n}-\frac{1}{2}}
\end{aligned}
$$

Next we show that the structure of the Bogolyubov coefficients does not change depending on how many 
sudden transitions there are between the first and the last period of constant $\epsilon$. For a sudden transition between two decelerating periods, from $\epsilon_{0}$ to $\epsilon_{1}$, we can calculate the partial Bogolyubov coefficients defined in (54) and (55),

$$
\begin{aligned}
\alpha_{1,0}(k)= & i A_{1,0} k^{-\nu_{0}-\nu_{1}}-i e^{i \pi \nu_{0}} B_{1,0} k^{\nu_{0}-\nu_{1}}-i e^{-i \pi \nu_{1}} C_{1,0} k^{-\nu_{0}+\nu_{1}} \\
& +i e^{i \pi\left(\nu_{0}-\nu_{1}\right)} D_{1,0} k^{\nu_{0}+\nu_{1}} \\
\beta_{1,0}(k)= & i A_{1,0} k^{-\nu_{0}-\nu_{1}}-i e^{i \pi \nu_{0}} B_{1,0} k^{\nu_{0}-\nu_{1}}-i e^{i \pi \nu_{1}} C_{1,0} k^{-\nu_{0}+\nu_{1}} \\
& +i e^{i \pi\left(\nu_{0}+\nu_{1}\right)} D_{1,0} k^{\nu_{0}+\nu_{1}}
\end{aligned}
$$

where

$$
\begin{aligned}
& A_{1,0}=M_{0}^{\prime}\left(\mathcal{H}_{0}\right) M_{1}\left(\mathcal{H}_{0}\right)-M_{0}\left(\mathcal{H}_{0}\right) M_{1}^{\prime}\left(\mathcal{H}_{0}\right), \\
& B_{1,0}=P_{0}^{\prime}\left(\mathcal{H}_{0}\right) M_{1}\left(\mathcal{H}_{0}\right)-P_{0}\left(\mathcal{H}_{0}\right) M_{1}^{\prime}\left(\mathcal{H}_{0}\right), \\
& C_{1,0}=M_{0}^{\prime}\left(\mathcal{H}_{0}\right) P_{1}\left(\mathcal{H}_{0}\right)-M_{0}\left(\mathcal{H}_{0}\right) P_{1}^{\prime}\left(\mathcal{H}_{0}\right), \\
& D_{1,0}=P_{0}^{\prime}\left(\mathcal{H}_{0}\right) P_{1}\left(\mathcal{H}_{0}\right)-P_{0}\left(\mathcal{H}_{0}\right) P_{1}^{\prime}\left(\mathcal{H}_{0}\right) .
\end{aligned}
$$

If the state is a $\mathrm{BD}$ one during the $\epsilon_{0}$-period, the full Bogolyubov coefficients correspond to the partial ones for one transition given in Eqs. (63)-(64).

For two successive transitions between decelerating periods we have from (52)

$$
\begin{aligned}
\alpha_{2,0}(k)= & \alpha_{2,1}(k) \alpha_{1,0}(k)+\beta_{2,1}^{*}(k) \beta_{1,0}(k) \\
= & i A_{2,0} k^{-\nu_{0}-\nu_{2}}-i e^{i \pi \nu_{0}} B_{2,0} k^{\nu_{0}-\nu_{2}}-i e^{-i \pi \nu_{2}} C_{2,0} k^{-\nu_{0}+\nu_{2}} \\
& +i e^{i \pi\left(\nu_{0}-\nu_{2}\right)} D_{2,0} k^{\nu_{0}+\nu_{2}}, \\
\beta_{2,0}(k)= & \beta_{2,1}(k) \alpha_{1,0}(k)+\alpha_{2,1}^{*}(k) \beta_{1,0}(k) \\
= & i A_{2,0} k^{-\nu_{0}-\nu_{2}}-i e^{i \pi \nu_{0}} B_{2,0} k^{\nu_{0}-\nu_{2}}-i e^{i \pi \nu_{2}} C_{2,0} k^{-\nu_{0}+\nu_{2}} \\
& +i e^{i \pi\left(\nu_{0}+\nu_{2}\right)} D_{2,0} k^{\nu_{0}+\nu_{2}},
\end{aligned}
$$

where

$$
\begin{aligned}
& A_{2,0}=2 \sin \left(\pi \nu_{1}\right)\left[B_{2,1} A_{1,0}-A_{2,1} C_{1,0}\right], \\
& B_{2,0}=2 \sin \left(\pi \nu_{1}\right)\left[B_{2,1} B_{1,0}-A_{2,1} D_{1,0}\right], \\
& C_{2,0}=2 \sin \left(\pi \nu_{1}\right)\left[D_{2,1} A_{1,0}-C_{2,1} C_{1,0}\right], \\
& D_{2,0}=2 \sin \left(\pi \nu_{1}\right)\left[D_{2,1} B_{1,0}-C_{2,1} D_{1,0}\right],
\end{aligned}
$$

or, when written in a matrix form,

$$
\begin{aligned}
\left(\begin{array}{ll}
B_{2,0} & A_{2,0} \\
D_{2,0} & C_{2,0}
\end{array}\right)= & 2 \sin \left(\pi \nu_{1}\right)\left(\begin{array}{ll}
B_{2,1} & A_{2,1} \\
D_{2,1} & C_{2,1}
\end{array}\right) \cdot\left(\begin{array}{cc}
1 & 0 \\
0 & -1
\end{array}\right) \\
& \cdot\left(\begin{array}{ll}
B_{1,0} & A_{1,0} \\
D_{1,0} & C_{1,0}
\end{array}\right) .
\end{aligned}
$$

Upon comparing the structure of Eqs. (63)-(64) with (69)-(70) we see that sudden matchings do not change the structure of the powers of the momentum in the deep IR. If there are $n$ sudden transitions between decelerating periods, the leading power of the momentum in the IR is $k^{-\nu_{0}-\nu_{n}}$. The same conclusion stands if some of the intermediary periods are accelerating ones, it is just that the coefficient of the leading term changes, but it is straightforward to calculate it. In the case when one considers many transitions it is convenient to use the suitably generalized matrix form (75), which for $n$ matchings becomes

$$
\begin{aligned}
\left(\begin{array}{ll}
B_{n, 0} & A_{n, 0} \\
D_{n, 0} & C_{n, 0}
\end{array}\right)= & {\left[\prod_{j=1}^{n-1} 2 \sin \left(\pi \nu_{j}\right)\left(\begin{array}{cc}
B_{j+1, j} & -A_{j+1, j} \\
D_{j+1, j} & -C_{j+1, j}
\end{array}\right)\right] } \\
& \cdot\left(\begin{array}{ll}
B_{1,0} & A_{1,0} \\
D_{1,0} & C_{1,0}
\end{array}\right) .
\end{aligned}
$$

These coefficients contain many terms with different powers of the Hubble rates at points of transition. If there exist a clear hierarchy between these Hubble rates many of the terms can be neglected as subdominant ones, and coefficients simplify significantly.

\section{CALCULATING THE IR OF INTEGRALS $\mathcal{I}_{s}$}

In this section we approximate and calculate integrals (48). Since the integration range here is $\left(k_{0}, \mu\right)$, and $k_{0} \ll$ $\mathcal{H}, \mathcal{H}_{0} \ll \mu \ll \mathcal{H}_{2} \ll \mathcal{H}_{1}$ (see Fig. 2), the ratios $k / \mathcal{H}_{1}$ and $k / \mathcal{H}_{2}$ are very small, and we expand parts of the Bogolyubov coefficients in powers of this ratio, keeping only the leading term. In the end this corresponds to expanding the full result in powers of $\left(\mathcal{H}, \mathcal{H}_{0}\right) /\left(\mathcal{H}_{1}, \mathcal{H}_{2}\right)$.

Bogolyubov coefficients can be written in terms of partial ones as as

$$
\begin{aligned}
& \alpha_{3,0}=\alpha_{3,1} \alpha_{1,0}+\beta_{3,1}^{*} \beta_{1,0}, \\
& \beta_{3,0}=\alpha_{3,1}^{*} \beta_{1,0}+\beta_{3,1} \alpha_{1,0} .
\end{aligned}
$$

The dependence on $k$ in $\alpha_{3,1}$ and $\beta_{3,1}$ appears only as $k / \mathcal{H}_{1}$ and $k / \mathcal{H}_{2}$, which means that we can expand these, keeping only the leading order contribution, which is, as derived in Sec. V B

$$
\alpha_{3,1}=\beta_{3,1}=\frac{i A_{3,1}}{k^{\nu_{I}+\nu_{M}}}=-\beta_{3,1}^{*},
$$

where $A_{3,1}$ is defined as in (76). In this particular case it is

$$
\begin{aligned}
A_{3,1}= & -\frac{1}{\pi} 2^{\nu_{I}-\frac{5}{2}} \Gamma\left(\nu_{I}\right) \Gamma\left(\nu_{M}\right)\left(1-\epsilon_{I}\right)^{\nu_{I}+\frac{1}{2}}\left(\nu_{I}-\frac{1}{2}\right) \\
& \times\left(\nu_{M}+\frac{3}{2}\right) \mathcal{H}_{1}^{\nu_{I}+\frac{1}{2}} \mathcal{H}_{2}^{\nu_{M}-\frac{1}{2}}
\end{aligned}
$$

to leading order in the ratio $\mathcal{H}_{2} / \mathcal{H}_{1} \ll 1$. This allows us to write the full coefficients as 


$$
\alpha_{3,0}=\beta_{3,0}=\beta_{3,1}\left(\alpha_{1,0}-\beta_{1,0}\right) .
$$

The fact that, to leading order, $\alpha_{3,0}=\beta_{3,0}$ simplifies the form of the integrand function,

$$
\begin{aligned}
Z_{\mathrm{Bog}}(k, \eta) & =4\left|\beta_{3,0}(k)\right|^{2} \Re^{2}\left[u_{M}(k, \eta)\right] \\
& =\frac{4\left|A_{3,1}\right|^{2}}{k^{2 \nu_{I}+2 \nu_{M}}}\left|\alpha_{1,0}-\beta_{1,0}\right|^{2} \Re^{2}\left[u_{M}(k, \eta)\right] .
\end{aligned}
$$

This expression further simplifies if we write out $\alpha_{1,0}$ and $\beta_{1,0}$ in terms of the BD mode functions, using the fact that $u_{R}^{\prime}(k, \eta)=-i k u_{R}(k, \eta)$,

$$
\left|\alpha_{1,0}(k)-\beta_{1,0}(k)\right|^{2}=\frac{2}{k}\left[\Re^{2}\left[u_{I}^{\prime}\left(k, \eta_{0}\right)\right]+k^{2} \Re^{2}\left[u_{I}\left(k, \eta_{0}\right)\right]\right],
$$

which can be rewritten using the equation of motion as

$$
\begin{aligned}
& \left|\alpha_{1,0}(k)-\beta_{1,0}(k)\right|^{2} \\
& \quad=\frac{2}{k}\left[\frac{1}{2} \frac{\partial^{2}}{\partial \eta_{0}^{2}}+2 k^{2}+f_{I}\left(\eta_{0}\right)\right] \Re^{2}\left[u_{I}\left(k, \eta_{0}\right)\right],
\end{aligned}
$$

where

$$
f_{I}\left(\eta_{0}\right)=-(1-6 \xi)\left(2-\epsilon_{I}\right) \mathcal{H}_{0}^{2}
$$

This gives us a very convenient representation for the leading order term of the integrand

$$
\begin{aligned}
Z_{\mathrm{Bog}}(k, \eta)= & \frac{8\left|A_{3,1}\right|^{2}}{k^{1+2 \nu_{I}+2 \nu_{M}}}\left[\frac{1}{2} \frac{\partial^{2}}{\partial \eta_{0}^{2}}+2 k^{2}+f_{I}\left(\eta_{0}\right)\right] \\
& \times \Re^{2}\left[u_{I}\left(k, \eta_{0}\right)\right] \Re^{2}\left[u_{M}(k, \eta)\right] .
\end{aligned}
$$

The integral over the integrand above is IR finite and we set $k_{0} \rightarrow 0$ right away (the terms that cancel the IR divergences from the $\mathrm{BD}$ contribution to energy density and pressure come from the subleading contributions in the expansion in ratios of Hubble rates).

The derivatives with respect to $\eta_{0}$ can be taken out of the integral, and we have for the $\mathcal{I}_{s}$ integrals

$$
\mathcal{I}_{s}^{(0, \mu)}=8\left|A_{3,1}\right|^{2}\left\{2 \mathcal{J}_{s+1}+\left[\frac{1}{2} \frac{\partial^{2}}{\partial \eta_{0}^{2}}+f_{I}\left(\eta_{0}\right)\right] \mathcal{J}_{s}\right\}
$$

where the $\mathcal{J}_{s}$ integrals are defined to be

$$
\begin{aligned}
\mathcal{J}_{s}= & \int_{0}^{\mu} d k k^{2 s-1-2 \nu_{I}-2 \nu_{M}} \Re^{2}\left[u_{I}\left(k, \eta_{0}\right)\right] \Re^{2}\left[u_{M}(k, \eta)\right] \\
= & \frac{\pi^{2}}{8\left(1-\epsilon_{I}\right) \mathcal{H}_{0} \mathcal{H}} \int_{0}^{\mu} d k k^{2 s-1-2 \nu_{I}-2 \nu_{M}} \\
& \times J_{\nu_{I}}^{2}\left(\frac{k}{\left(1-\epsilon_{I}\right) \mathcal{H}_{0}}\right) J_{\nu_{M}}^{2}\left(\frac{2 k}{\mathcal{H}}\right), \quad s=1,2,3 .
\end{aligned}
$$

As they stand, $\mathcal{J}_{s}$ integrals cannot be solved analytically, because of the finite upper limit of integration $\mu$. But we are not interested in the dependence on $\mu$, since this is just a fiducial scale we have introduced to facilitate the approximation of the full integral. What we are interested in is extracting the $\mu$-independent contribution, which correspond to expanding the integrals above in powers of $1 / \mu$. ${ }^{4}$ The leading order term is $(1 / \mu)^{0}$, and the $\mu$-dependent terms should cancel against the terms coming from the UV, order by order. In practice this cancellation is hard to prove exactly since different approximations are used to calculate the UV and the IR, and all orders should be calculated explicitly.

The leading order contribution to integrals (88) corresponds to extending the limit of integration $\mu \rightarrow \infty$. Again, this does not correspond to capturing the UV contribution correctly (which we argued is irrelevant), but rather to a leading approximation for the IR contribution. These integrals exist, and for $\mathcal{H}>2\left(1-\epsilon_{I}\right) \mathcal{H}_{0}$ they are

$$
\begin{aligned}
\mathcal{J}_{s} \approx & \frac{2^{-3-2 s+2 \nu_{I}+2 \nu_{M}} \sqrt{\pi} \Gamma\left(s-\frac{1}{2}-\nu_{I}\right) \Gamma\left(1-s+\nu_{I}+\nu_{M}\right)}{\Gamma\left(\frac{3}{2}-s+\nu_{I}+\nu_{M}\right) \Gamma\left(\frac{3}{2}-s+\nu_{I}+2 \nu_{M}\right)} \mathcal{H}^{-2+2 s-2 \nu_{I}-2 \nu_{M}} \\
& \times{ }_{4} F_{3}\left(\frac{1}{2}, \frac{1}{2}-\nu_{I}, \frac{1}{2}+\nu_{I}, 1-s+\nu_{I}+\nu_{M} ; \frac{3}{2}-s+\nu_{I}, \frac{3}{2}-s+\nu_{I}+\nu_{M}, \frac{3}{2}-s+\nu_{I}+2 \nu_{M} ; \frac{4\left(1-\epsilon_{I}\right)^{2} \mathcal{H}_{0}^{2}}{\mathcal{H}^{2}}\right) \\
& +\frac{\pi^{3 / 2} \Gamma(s) \Gamma\left(\frac{1}{2}-s+\nu_{I}\right)}{16 \Gamma^{2}\left(1+\nu_{M}\right) \Gamma\left(1-s+\nu_{I}\right) \Gamma\left(1-s+2 \nu_{I}\right)}\left[\left(1-\epsilon_{I}\right) \mathcal{H}_{0}\right]^{-1+2 s-2 \nu_{I} \mathcal{H}^{-1-2 \nu_{M}}} \\
& \times{ }_{4} F_{3}\left(s, s-2 \nu_{I}, s-\nu_{I}, \frac{1}{2}+\nu_{M} ; \frac{1}{2}+s-\nu_{I}, 1+\nu_{M}, 1+2 \nu_{M} ; \frac{4\left(1-\epsilon_{I}\right)^{2} \mathcal{H}_{0}^{2}}{\mathcal{H}^{2}}\right),
\end{aligned}
$$

\footnotetext{
${ }^{4}$ Formally, one can evaluate $\mathcal{J}_{s}$ integrals by writing them as $\int_{0}^{\mu}=\int_{0}^{\infty}-\int_{\mu}^{\infty}$. The former integral contains the leading ( $\mu$-independent) contribution, while the latter integral yields an asymptotic series in powers of $1 / \mu$. The hope is that this series can be (Borrel) resummed, yielding an expression that gets canceled by the $\mu$-dependent UV contribution.
} 
and for $\mathcal{H}<2\left(1-\epsilon_{I}\right) \mathcal{H}_{0}$

$$
\begin{aligned}
\mathcal{J}_{s} \approx & \frac{\sqrt{\pi} \Gamma\left(s-\frac{1}{2}-\nu_{M}\right) \Gamma\left(1-s+\nu_{I}+\nu_{M}\right)}{32 \Gamma\left(\frac{3}{2}-s+\nu_{I}+\nu_{M}\right) \Gamma\left(\frac{3}{2}-s+2 \nu_{I}+\nu_{M}\right)}\left[\left(1-\epsilon_{I}\right) \mathcal{H}_{0}\right]^{-2+2 s-2 \nu_{I}-2 \nu_{M}} \\
& \times{ }_{4} F_{3}\left(\frac{1}{2}, \frac{1}{2}-\nu_{M}, \frac{1}{2}+\nu_{M}, 1-s+\nu_{I}+\nu_{M} ; \frac{3}{2}-s+\nu_{M}, \frac{3}{2}-s+\nu_{I}+\nu_{M}, \frac{3}{2}-s+2 \nu_{I}+\nu_{M} ; \frac{\mathcal{H}^{2}}{4\left(1-\epsilon_{I}\right)^{2} \mathcal{H}_{0}^{2}}\right) \\
& +\frac{2^{-4-2 s-2 \nu_{I}+2 \nu_{M}} \pi^{3 / 2} \Gamma(s) \Gamma\left(\frac{1}{2}-s+\nu_{M}\right)}{\Gamma^{2}\left(1+\nu_{I}\right) \Gamma\left(1-s+\nu_{M}\right) \Gamma\left(1-s+2 \nu_{M}\right)}\left[\left(1-\epsilon_{I}\right) \mathcal{H}_{0}\right]^{-1-2 \nu_{I}} \mathcal{H}^{-1+2 s-2 \nu_{M}} \\
& \times{ }_{4} F_{3}\left(s, \frac{1}{2}+\nu_{I}, s-2 \nu_{M}, s-\nu_{M} ; 1+\nu_{I}, 1+2 \nu_{I}, \frac{1}{2}+2-\nu_{M} ; \frac{\mathcal{H}^{2}}{4\left(1-\epsilon_{I}\right)^{2} \mathcal{H}_{0}^{2}}\right),
\end{aligned}
$$

where ${ }_{4} F_{3}$ is the generalized hypergeometric function. These two solutions on two different regions actually represent one continuous solution, which we were not able to write as one function over the whole interval. Integrals $\mathcal{I}$ follow from these via expression (87). The derivatives with respect to $\eta_{0}$ can be replaced by more convenient ones with respect to $\mathcal{H}_{0}$. Remember that derivatives with respect to $\eta_{0}$ are actually derivatives of $u_{I}(k, \eta)$ evaluated at $\eta_{0}$. Since $u_{I}$ depends on conformal time only via $\mathcal{H}$ we can exploit the chain rule,

$$
\frac{\partial}{\partial \eta}=\mathcal{H}^{\prime} \frac{\partial}{\partial \mathcal{H}}=\left(1-\epsilon_{I}\right) \mathcal{H}^{2} \frac{\partial}{\partial \mathcal{H}},
$$

and evaluate derivatives at $\eta_{0}$ to yield

$$
\begin{aligned}
\mathcal{I}_{s} \approx \mathcal{I}_{s}^{(0, \mu)}= & 8\left|A_{3,1}\right|^{2}\left\{2 \mathcal{J}_{s+1}+\left[\frac{1}{2}\left(1-\epsilon_{I}\right)^{2} \mathcal{H}_{0}^{4} \frac{\partial^{2}}{\partial \mathcal{H}_{0}^{2}}\right.\right. \\
& \left.\left.+\left(1-\epsilon_{I}\right)^{2} \mathcal{H}_{0}^{3} \frac{\partial}{\partial \mathcal{H}_{0}}-(1-6 \xi)\left(2-\epsilon_{I}\right) \mathcal{H}_{0}^{2}\right] \mathcal{J}_{s}\right\}
\end{aligned}
$$

\section{ENERGY DENSITY AND PRESSURE IN MATTER ERA}

Energy density and pressure follow from $\mathcal{I}$ integrals given in (92) via (42) and (43). Derivatives with respect to $\eta$ there can be replaced by more convenient derivatives with respect to $\mathcal{H}$ [utilizing (91) again],

$$
\begin{gathered}
\rho_{q}=\frac{\hbar}{c^{3}} \times \frac{1}{4 \pi^{2} a^{4}}\left\{2 \mathcal{I}_{2}+\frac{1}{2}\left[(1-6 \xi) \mathcal{H}^{2}\right.\right. \\
\left.\left.+\frac{3}{2}(1-4 \xi) \mathcal{H}^{3} \frac{\partial}{\partial \mathcal{H}}+\frac{1}{4} \mathcal{H}^{4} \frac{\partial^{2}}{\partial \mathcal{H}^{2}}\right] \mathcal{I}_{1}\right\}, \\
p_{q}=\frac{\hbar}{c^{3}} \times \frac{1}{4 \pi^{2} a^{4}}\left\{\frac{2}{3} \mathcal{I}_{2}+\frac{1}{2}\left[(1-6 \xi) \mathcal{H}^{2}\right.\right. \\
\left.\left.+\frac{1}{2}(3-16 \xi) \mathcal{H}^{3} \frac{\partial}{\partial \mathcal{H}}+\frac{1}{4} \mathcal{H}^{4}(1-4 \xi) \frac{\partial^{2}}{\partial \mathcal{H}^{2}}\right] \mathcal{I}_{1}\right\},
\end{gathered}
$$

where we have restored the units. By taking the limit of these expressions $\epsilon_{I} \rightarrow 0, \xi \rightarrow 0, \mathcal{H}_{0} \rightarrow 0$ we precisely recover the main result of [1] where the backreaction scales just as the background during matter period. In order to evaluate these expressions in a more general setting presented here, we have to specify all the parameters $\mathcal{H}_{0}, \mathcal{H}_{1}, \mathcal{H}_{2}, \epsilon_{I}$. These have to be connected with the usual cosmological parameters which were taken from [33].

The value of the Hubble rate today is $\mathcal{H}=H=68 \frac{\mathrm{km}}{\mathrm{Mpcs}}$ (for $a=1$ today). The redshift of radiation-matter equality $z_{\text {eq }}=3270$ fixes the duration of the matter period, and hence $\mathcal{H}_{2}$. The requirement of at least minimal duration of inflationary period, namely $\mathcal{H}_{0}=\mathcal{H}$ and the amplitude of the scalar perturbations of the cosmic microwave background $(\mathrm{CMB})$ fix the duration of inflation and radiation period, up to $\epsilon_{I}$. That is fixed by the tensor to scalar perturbation ratio in the $\mathrm{CMB}$ which was claimed to be measured recently $(r=0.2[3])$ giving $\epsilon_{I}=0.01$. How $\mathcal{H}_{i}$ are determined from these quantities is given in Appendix B. The parameters that are left unspecified (whose parameter space we will explore) are the duration of inflation, expressed in its number of $e$-foldings $N_{I}$, and the nonminimal coupling $\xi$.

Few plots of the backreaction during matter era for different parameters $\left(N_{I}, \xi\right)$ are presented in Fig. 3 to give a feeling for the dependence on parameters. They all show transient behavior in a form of a pronounced peak of the ratio $\rho_{q} / \rho_{b}$ around the time when $\mathcal{H}$ becomes comparable to $\mathcal{H}_{0}$. The amplitude of the effect is very sensitive to changing $\xi$. Changing $N_{I}$ modifies the amplitude somewhat, but also moves the onset of the transient effect, since it changes the time when $\mathcal{H} \sim \mathcal{H}_{0}$.

Obviously, there is little point considering parameters for which the backreaction is always a few orders of magnitude smaller than the background. But, also, there is no point in examining the behavior of backreaction during matter period when it is much larger than the background. That signals it must have become important much before the onset of matter period. That gives us a way to constrain our parameters. First of all, we require the backreaction to be 

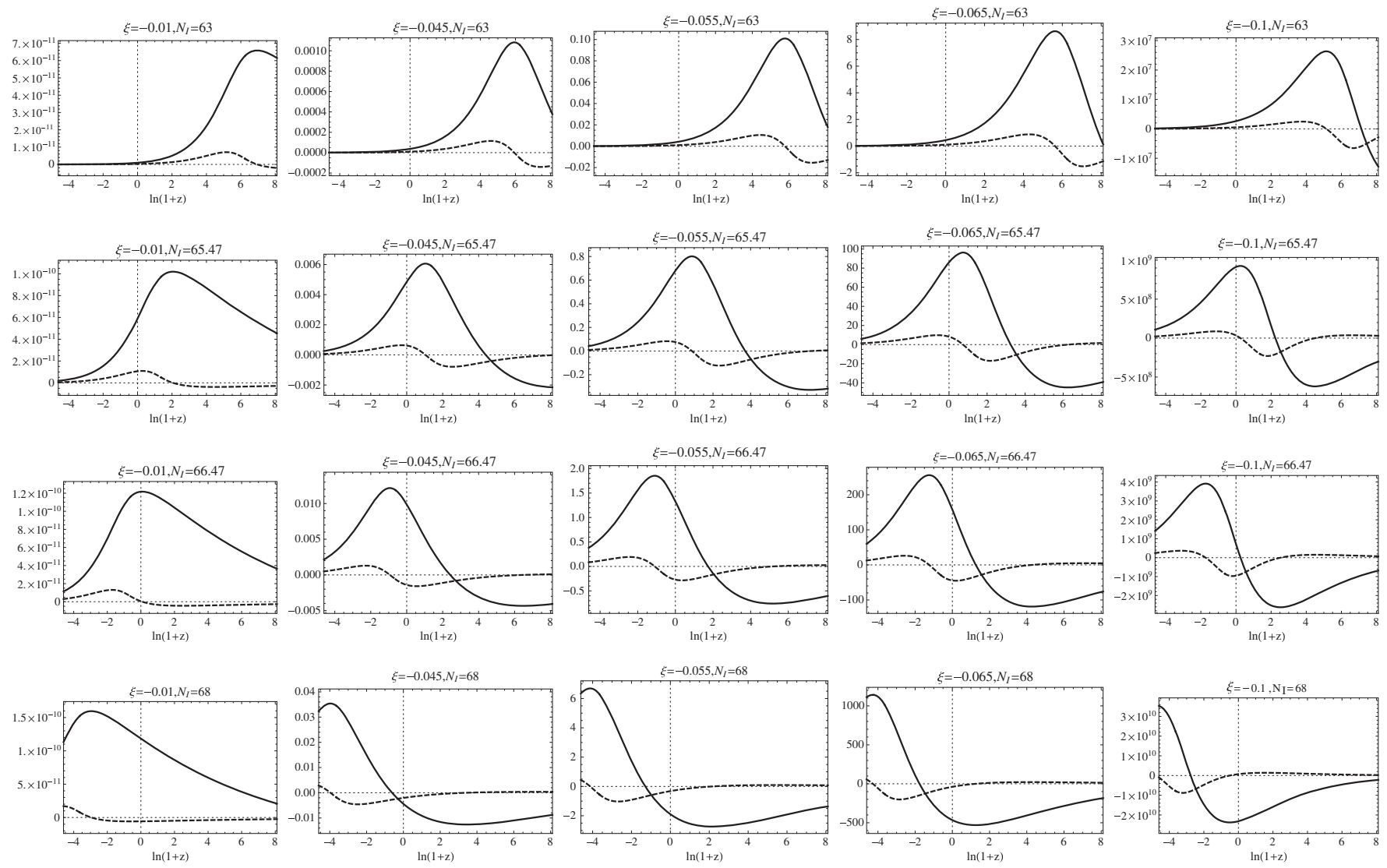

FIG. 3. The backreaction during matter era depending on nonminimal coupling $\xi$ and number of $e$-foldings of inflation $N_{I}$. Solid curves represent the ratio $\rho_{q} / \rho_{b}$, and dashed curves ratio $p_{q} / \rho_{b}$ (vertical axis). The horizontal axis represents the time in terms of the logarithm of redshift. Redshift $z=0$ corresponds to today, larger values to the past, and negative values to the future.

small during inflation. The bound for this is $\rho_{q} / \rho_{b} \sim 1$ at the end of inflation. ${ }^{5}$ This ratio can be inferred from expression (59) of Ref. [8], and is plotted here in Fig. 4.

Even though studying strong backreaction during inflation is an interesting topic by itself, here we concentrate on studying backreaction that becomes strong only in late-time matter era. During radiation-dominated era the ratio $\rho_{q} / \rho_{b}$ does not change appreciably (that result is presented in Appendix C). If, for given $\xi$ and $N_{I}$ backreaction becomes non-negligible during matter period, but is still in perturbative range $\left(\rho_{q} / \rho_{b}<1\right)$, we can hope to get some intuition about its influence on the background evolution if we plot the perturbatively corrected $\epsilon$ parameter, which we infer from the perturbatively corrected Friedmann equations,

\footnotetext{
${ }^{5}$ Studying the parameter space for which backreaction becomes large during inflation would be interesting in its own right. Especially since in that case it exhibits the behavior of a perfect fluid with $w<-1$ and negative contribution to the energy density, which signals it would work towards slowing down inflation, and perhaps ending it. This possibility was examined in the case of scalars [12,15,34], and in the case of gravitons $[35,36]$. Here a full self-consistent (numerical) solution should be found to be sure how it influences the dynamics of the expansion (much like in [37]).
}

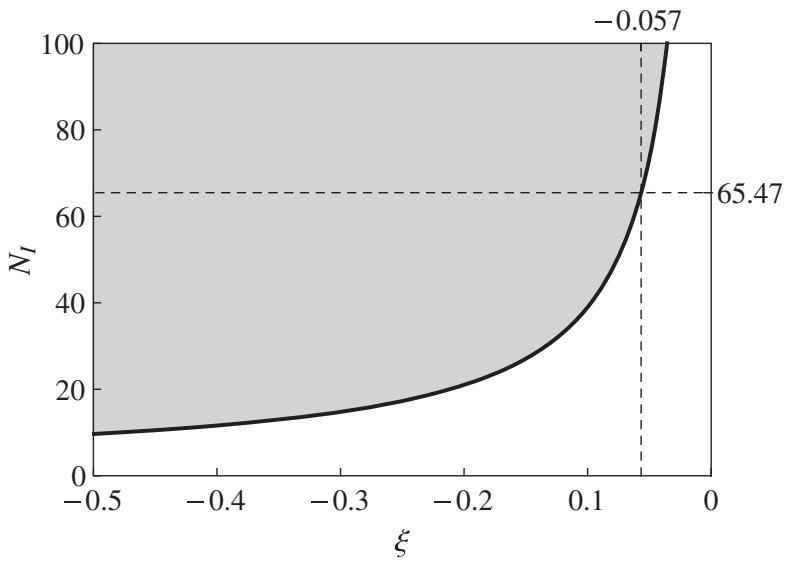

FIG. 4. The condition $\rho_{q} / \rho_{b}=1$ at the end of inflation depending on the nonminimal coupling and the number of $e$-foldings of inflation $N_{I}$. The shaded region represents the parameter space where this ratio is larger than 1 and where the backreaction influences the evolution during inflation. We do not study this case in this work. We study the unshaded region, above the dashed line of minimal inflation, $N_{I \text {, min }} \approx 65.47$ (taking the BICEP2 result seriously). This puts the constraint on nonminimal coupling interesting for this study to be $-0.057<\xi<0$. 


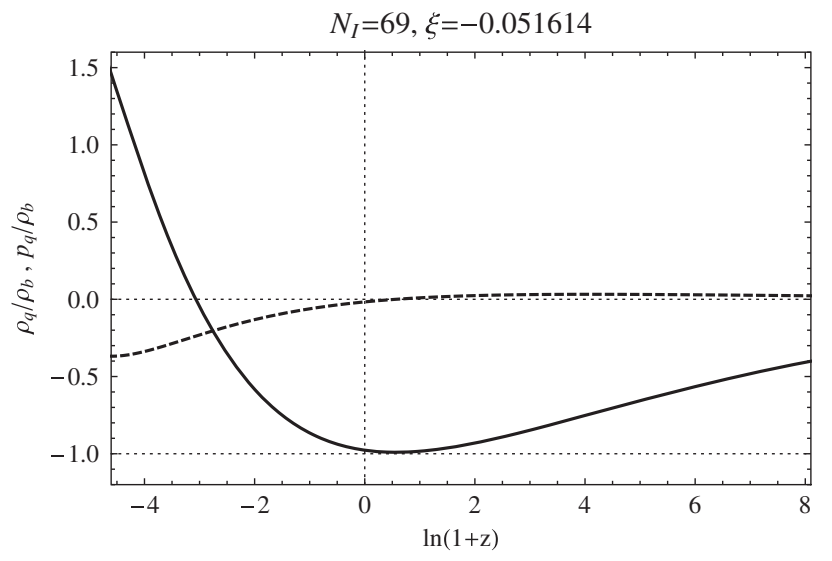

(a)

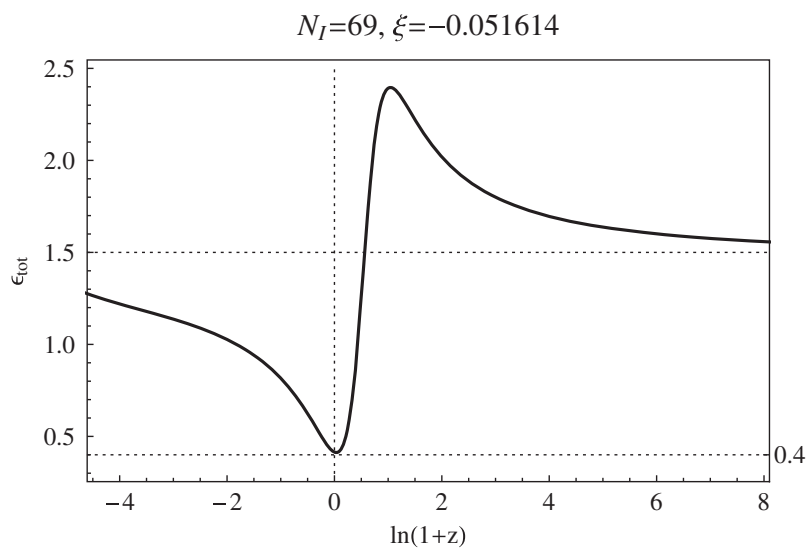

(b)

FIG. 5. The two plots show (a) ratio of the quantum energy density (bold) and quantum pressure (dashed) to the background energy density during matter dominated period, and (b) naively corrected $\epsilon$ parameter during matter period, both for choice of the number of $e$-foldings of inflation $N_{I}=69$ and nonminimal coupling $\xi=-0.051614$.

$$
\epsilon_{\mathrm{tot}}=1-\frac{\mathcal{H}^{\prime}}{\mathcal{H}^{2}}=\frac{3}{2}\left(1+\frac{p_{q}}{\rho_{b}+\rho_{q}}\right)
$$

There is (at least) one interesting choice of parameters, $\xi=-0.051614$ and $N_{I}=69$, for which we are on the very edge of perturbative range up until today, for which the $\epsilon$ parameter takes the value $\epsilon \sim 0.4$, as it is measured today. The energy density, pressure, and $\epsilon$ parameter in this case are shown in Fig. 5. For that choice of parameters at the end of inflation we have $\rho_{q} / \rho_{b} \sim-0.39$, and during radiation $\rho_{q} / \rho_{b} \sim-0.43$ which is mildly inside the perturbative regime, but still large enough to affect the background evolution.

Of course, this estimate should not be taken too seriously. Backreaction in fact becomes too large to naively use (95). It predicts that $\epsilon$ changes so much primarily because $\rho_{q}$ comes very close to $-\rho_{b}$ (but the direction of change is primarily determined by the sign of the quantum fluid pressure). This means that the Hubble rate must decrease considerably [which can be inferred from the first Friedmann equation (2)], which is in disagreement with the value measured today that we have assumed. In order to find the true behavior, one would have to solve the one-loop quantum corrected Friedmann equations self-consistently, much like in [37] (which is the only such study we are aware of). Nevertheless, this particular example indicates a very interesting possibility that this model, although finely tuned, could account for the late-time acceleration of the Universe (or other interesting possible effects), and certainly provides good motivation for further investigation.

\section{CONCLUSIONS}

In this paper we study the one-loop quantum backreaction of a nonminimally coupled massless scalar field (with vanishing expectation value) on a universe that goes through a series of constant $\epsilon$ eras connected by fast transitions. In our model the universe starts in a radiation era, which is followed by a period of inflation, radiation and finally a matter era (Fig. 1). We assume a fixed, flat, FLRW background metric and compute the one-loop expectation value of the energy-momentum tensor for our scalar in order to estimate its effect on the background evolution. Our model is strictly speaking predictive only when the quantum fluid is subdominant to the background. When the quantum backreaction becomes comparable to the background, a self-consistent solution has to be found by performing a self-consistent treatment of the background equation in presence of the (one-loop) quantum fluctuations. Such a treatment is left for future work; here we determine in which cases the self-consistent treatment might yield interesting predictions.

Our main results and observations can be summarized as follows:

(i) When the nonminimal coupling is negative, the quantum backreaction is negative and, for a sufficiently large negative coupling, it grows with respect to the background, becoming eventually dominant (see e.g. [8]). This latter case can be treated by solving self-consistently the equations of semiclassical gravity. For a minimal inflation with $N_{\text {efolds }} \sim 66$, the value of $\xi$ for which the backreaction becomes significant at the end of inflation is about $\xi \simeq-0.057$. It is plausible that such a backreaction would provide a graceful exit from inflation without having to resort to fine tuning of the inflaton potential (such to give a vanishing contribution to the cosmological constant) at the end of inflation. This question deserves further investigation. Furthermore, studying quantum backreaction during inflation can be used to constrain the physically acceptable values of nonminimal coupling for light scalar fields during inflation (adiabatic inflaton condensates such as the Higgs field of Higgs 
inflation are exempt from this constraint and they require a separate analysis).

(ii) Similarly as found in the case of a minimally coupled scalar [1], during radiation era the backreaction contribution of a nonminimally coupled scalar does not change significantly when compared with the background. This also means that, if the backreaction is subdominant at the end of inflation, it will remain so at the beginning of matter era.

(iii) When $\xi<-1 / 3$, the relative quantum backreaction during matter era is negative and it grows. However, for realistic duration of inflation $\left(N_{I}>65\right)$, it already dominates during inflation, making this case physically irrelevant. One can nevertheless construct a viable (albeit more complex) model in this case, by making $\xi$ field dependent, $\xi=\xi(\chi)(\chi$ symbolizing some other field $\phi$ is coupled to), and arranging it such that during inflation $\xi$ is above the critical value (for which quantum backreaction remains subdominant throughout inflation), and $\xi<-1 / 3$ during matter era, such that the relative backreaction grows in matter era. It would be of interest to study the selfconsistent evolution of the Universe in this case. Namely, a negative backreaction slows down the rate of Universe's expansion, and in the extreme case when it is large and negative, it could even reverse the expansion to a contraction, thus completely changing the future of our Universe.

(iv) A potentially very interesting transient occurs for inflation close to minimal $\left(N_{\text {efolds }} \sim 69\right)$ and the nonminimal coupling slightly larger than its critical value, $\xi \sim-0.052 \gtrsim \xi_{c} \simeq-0.057$. In this case, the quantum backreaction exhibits a transient behavior in recent times (illustrated in Fig. 5), and changes from negative to positive. This case bears resemblance to late-time dark energy, and a self-consistent treatment of semiclassical gravity is needed in order to find out whether this is a good model for dark energy. Since there are essentially no free parameters in this model (the values of the number of $e$-folds and the nonminimal coupling are to a large extent fixed by the requirement that the transient occurs in recent times and by the requirement that the backreaction reaches a maximum value that is comparable to the background energy density), this model can be easily tested by astronomical observations of the Hubble rate as a function of the redshift, and by studying late-time evolution of small perturbations on top of the homogeneous background. These studies are however beyond of the scope of this work.

(v) In the limit when $\xi \rightarrow 0$ (minimal coupling) and $\mathcal{H}_{0} \rightarrow 0$ (very long inflation), our results agree with those of [1]. In particular, by taking a careful limit $\xi \rightarrow 0$, we find the term in the relative quantum backreaction that grows logarithmically in time during radiation (Appendix $\mathrm{C}$ ), that is absent in the case when $\xi<0$.

(vi) Our late-time quantum backreaction is dominated by the infrared fluctuations, and does not depend on details of the transitions, provided the transitions are fast (faster than the Hubble rate at the relevant transition). However, if any of the transitions is slow (a slow transition can be modeled by e.g. a tangent hyperbolic change of the $\epsilon$ parameter, or as was done in [38]), the leading order late-time quantum backreaction will in general acquire a dependence on the transition rate. In this case we expect a reduced amplitude of the late-time backreaction, but an identical scaling in time. Hence, including the case of slow transition(s) is inessential, and we will not study it further here.

(vii) It would be of interest to extend our analysis to inflationary fluctuations of other quantum fields. Gravitons were already studied in [39] and [1], and their contribution scales as matter in matter era, and constitute a small contribution to dark matter. A heuristic analysis suggests that a similar conclusion can be reached for adiabatic scalar cosmological perturbations [1], although the case of a nonminimally coupled inflaton is more delicate and it requires a separate investigation.

(viii) Naively, one might expect no significant quantum backreaction from massless gauge fields such as photons. Indeed, at the classical level, photons couple conformally to gravity and hence their quantum backreaction can be treated as that of any conformally coupled field. The quantum backreaction of conformal fields can be represented by local higher-derivative curvature terms, and a general study [40] shows that their late-time backreaction is completely negligible. However, when one includes couplings of gauge fields to other fields such as (light) massless scalars, a significant photon production during inflation is in fact possible [41,42], making a detailed study of the late-time backreaction from gauge fields worthwhile.

(ix) One might think that the Pauli exclusion principle will prevent fermions from giving a large quantum backreaction (because population of the infrared states is limited to one fermion per state). This is however not true, since fermion loops contribute with an opposite sign, and hence can destabilize the vacuum of a scalar field in an expanding space-time (for a study of a Yukawa theory on de Sitter case see Ref. [23]). This suggests that studying late-time quantum backreaction from fermions could yield interesting results for cosmology.

(x) One might argue that the late-time backreaction we calculate here is unobservable, since it primarily 
comes from super-Hubble modes. This is however not true for the following reasons. Firstly, the oneloop stress energy tensor we calculate is gauge invariant. ${ }^{6}$ And secondly, its value is invariant under spatial translations (this immediately follows from the fact that $T_{\mu \nu}^{q}$ depends on time but it is independent of space), and hence each local (freely falling) observer on a FLRW background will agree on the value of the backreaction. Our calculation neglects small fluctuations on top of a homogeneous expanding background. In order to study how these fluctuations affect the result presented here, one would have to study $\left\langle\Omega\left|\hat{T}_{\mu \nu}(x) \hat{T}_{\rho \sigma}\left(x^{\prime}\right)\right| \Omega\right\rangle$, which is worth doing once one has a good candidate for dark energy.

\section{ACKNOWLEDGMENTS}

This work is part of the D-ITP consortium, a program of the Netherlands Organisation for Scientific Research (NWO) that is funded by the Dutch Ministry of Education, Culture and Science (OCW).

\section{APPENDIX A: RENORMALIZATION OF THE ENERGY-MOMENTUM TENSOR ON SMOOTH FLRW}

This appendix presents an outline of the regularization and renormalization procedures needed to assign finite values to expressions (24) and (25). The exposition is essentially the same as in Appendix A from [1], just extended to include nonminimal coupling. The material here is standard (see [43]), and we include it for the sake of notation and completeness. For a different method of regularization see [44]. We think that removing the dependence on the UV cutoff is essential for obtaining reliable results, since it can lead to some spurious time evolution as in the case of e.g. just introducing a comoving momentum cutoff [45], or introducing a physical momentum cutoff [46] which also leads to nonconservation of the energy-momentum tensor.

\footnotetext{
${ }^{6}$ In order to see that, note that our scalar field is a spectator field, and thus its expectation value is zero in all epochs, i.e. we have, $\hat{\Phi}(x)=\phi_{0}(t)+\hat{\varphi}(x)$, where $\langle\Omega|\hat{\Phi}(x)| \Omega\rangle=\phi_{0}(t) \rightarrow 0$. This then implies that the stress energy tensor is gauge invariant (to quadratic order in perturbations). This can be shown as follows. Under a coordinate transformation $x^{\mu} \rightarrow x^{\mu}+\xi^{\mu}(x)$, a scalar field transform as, $\Phi(x) \rightarrow \tilde{\Phi}(x)=\Phi(x)-\xi^{\mu} \partial_{\mu} \tilde{\Phi}(x)$ plus higher order terms. In the absence of condensate $\phi_{0}$, this reduces to $\varphi(x) \rightarrow$ $\tilde{\varphi}(x)=\varphi(x)-\xi^{\mu} \partial_{\mu} \varphi(x)$ plus cubic order terms (here we took $\xi^{\mu}$ and $\varphi$ to be first order quantities). The one-loop energy-momentum tensor can be represented as a differential operator acting on $\left\langle\Omega\left|\hat{\varphi}(x)^{2}\right| \Omega\right\rangle$, which to lowest order transforms as $\left\langle\Omega\left|\hat{\varphi}(x)^{2}\right| \Omega\right\rangle \rightarrow$ $\left\langle\Omega\left|\hat{\tilde{\varphi}}(x)^{2}\right| \Omega\right\rangle=\left\langle\Omega\left|\hat{\varphi}(x)^{2}\right| \Omega\right\rangle-\xi^{\mu} \partial_{\mu}\left\langle\Omega\left|\hat{\varphi}(x)^{2}\right| \Omega\right\rangle$ plus higher order terms. Notice that the leading correction is cubic in perturbations, and hence $\left\langle\Omega\left|\hat{\varphi}(x)^{2}\right| \Omega\right\rangle$ is gauge invariant to quadratic order in gauge transformations. which completes the proof.
}

Integrals in (24) and (25) are split into UV and IR parts by some constant cutoff scale $\mu \gg \mathcal{H}$. The IR parts can be evaluated in $D=4$ right away. The UV part needs to be regularized, and the method of choice is dimensional regularization. It entails evaluating the integrals in arbitrary $D$ dimensions for which the integrals converge. This automatically eliminates any power-law divergences in $D=4$, but not the logarithmic one, which in dimensional regularization presents itself as $1 /(D-4)$ term. That term is to be absorbed into the higher-derivative gravitational counterterms and after that the limit $D \rightarrow 4$ is to be taken. This entails renormalization after which we are left with a finite final answer for energy density and pressure.

The practical task is to isolate this $1 /(D-4)$ divergence and show that it can be absorbed into the counterterm. The integrals to evaluate are

$$
\begin{aligned}
\rho_{q}^{\mathrm{UV}, 0}= & \frac{a^{-D}}{(4 \pi)^{\frac{D-1}{2}} \Gamma\left(\frac{D-1}{2}\right)} \int_{\mu}^{\infty} d k k^{D-2} \\
& \times\left[2 k^{2}|u|^{2}-\frac{1}{2}[D-2-4 \xi(D-1)] \mathcal{H}^{\prime}|u|^{2}\right. \\
& \left.-\frac{1}{2}[D-2-4 \xi(D-1)] \mathcal{H} \frac{\partial}{\partial \eta}|u|^{2}+\frac{1}{2} \frac{\partial^{2}}{\partial \eta^{2}}|u|^{2}\right],
\end{aligned}
$$

$$
\begin{aligned}
p_{q}^{\mathrm{UV}, 0}= & \frac{a^{-D}}{(4 \pi)^{\frac{D-1}{2}} \Gamma\left(\frac{D-1}{2}\right)} \int_{\mu}^{\infty} d k k^{D-2} \\
& \times\left[\frac{2 k^{2}}{D-1}|u|^{2}-\frac{1}{2}[D-2-4 \xi(D-1)] \mathcal{H}^{\prime}|u|^{2}\right. \\
& -\frac{1}{2}[D-2-4 \xi(D-1)] \mathcal{H} \frac{\partial}{\partial \eta}|u|^{2} \\
& \left.+\frac{1}{2}(1-4 \xi) \frac{\partial^{2}}{\partial \eta^{2}}|u|^{2}\right] .
\end{aligned}
$$

This is to be done by finding the UV asymptotic expansion of the mode function, integrating term by term, keeping the terms that are divergent or finite in the limits $D \rightarrow 4$ and $\mu \rightarrow \infty$, and throwing away the ones that are zero in this limit.

The asymptotic expansion is obtained as a WentzelKramers-Brillouin approximation of the equation of motion (17), since $k \geq \mu \gg \mathcal{H}$. The shortcut way of doing this is to assume the positive-frequency expansion of $u$ in powers of $1 / k$,

$$
\begin{aligned}
u= & \frac{e^{-i k \eta}}{\sqrt{2 k}}\left[1+\frac{i F_{1}(\eta)}{k}+\frac{F_{2}(\eta)}{k^{2}}+\frac{i F_{3}(\eta)}{k}+\frac{F_{4}(\eta)}{k^{4}}\right] \\
& +\mathcal{O}\left(k^{-5}\right) .
\end{aligned}
$$

We have the freedom to multiply this mode function by a constant phase factor, but since it is physically irrelevant, 
here we chose the most convenient one for the discussion at hand. Now we can solve for $F_{n}(\eta)$ 's by solving the equation of motion (17) order by order in $1 / k$,

$$
\begin{gathered}
F_{1}^{\prime}=-\frac{1}{2} f, \\
F_{2}^{\prime}=\frac{1}{2}\left[F_{1}^{\prime \prime}+f F_{1}\right], \\
F_{3}^{\prime}=-\frac{1}{2}\left[F_{2}^{\prime \prime}+f F_{2}\right], \\
F_{4}^{\prime}=\frac{1}{2}\left[F_{3}^{\prime \prime}+f F_{3}\right],
\end{gathered}
$$

where $f$ is defined in (18), and the same for Wronskian normalization condition,

$$
\begin{gathered}
2 F_{2}+F_{1}^{2}-F_{1}^{\prime}=0, \\
2 F_{4}+2 F_{3} F_{1}+F_{2}^{2}-F_{3}^{\prime}+F_{2}^{\prime} F_{1}-F_{1} F_{2}=0 .
\end{gathered}
$$

Integrating equations (A4)-(A7) and imposing conditions (A8), (A9) yields

$$
\begin{gathered}
F_{1}(\eta)=-\frac{1}{2} \int_{\eta_{0}}^{\eta} d \tau f(\tau), \\
F_{2}(\eta)=-\frac{f(\eta)}{4}-\frac{1}{8}\left[\int_{\eta_{0}}^{\eta} d \tau f(\tau)\right]^{2},
\end{gathered}
$$

$$
\begin{aligned}
F_{3}(\eta)= & \frac{1}{8}\left[f^{\prime}(\eta)-f^{\prime}\left(\eta_{0}\right)\right]+\frac{f(\eta)}{8} \int_{\eta_{0}}^{\eta} d \tau f(\tau) \\
& +\frac{1}{48}\left[\int_{\eta_{0}}^{\eta} d \tau f(\tau)\right]^{3}+\frac{1}{8} \int_{\eta_{0}}^{\eta} d \tau f^{2}(\tau), \quad \text { (A12) } \\
F_{4}(\eta)= & \frac{f^{\prime \prime}(\eta)}{16}+\frac{5 f^{2}(\eta)}{32}+\frac{1}{16}\left[f^{\prime}(\eta)-f^{\prime}\left(\eta_{0}\right)\right] \int_{\eta_{0}}^{\eta} d \tau f(\tau) \\
& +\frac{f(\eta)}{32}\left[\int_{\eta_{0}}^{\eta} d \tau f(\tau)\right]^{2}+\frac{1}{384}\left[\int_{\eta_{0}}^{\eta} d \tau f(\tau)\right]^{4} \\
& +\frac{1}{16}\left[\int_{\eta_{0}}^{\eta} d \tau f(\tau)\right]\left[\int_{\eta_{0}}^{\eta} d \tau f^{2}(\tau)\right],
\end{aligned}
$$

where we have imposed the initial condition that all $F_{n}$ 's for odd $n$ 's are zero at $\eta_{0}$. This, again, is nothing other than picking an arbitrary phase.

An important thing to note here is that the time evolution of the mode function in the UV is adiabatic, it does not change its positive-frequency character. In other words, the evolution will induce no mode mixing in the UV to any adiabatic order, at most it will be suppressed to decay faster in the UV than any power of $1 / k$. This is quantified as a result on the Bogolyubov coefficients, $\alpha(k) \stackrel{k \rightarrow \infty}{\rightarrow} 1$ and $\beta(k) \stackrel{k \rightarrow \infty}{\rightarrow} 0$ faster than any power of $1 / k$.

Now it is a simple matter to evaluate (A1) and (A2), expand the result around $D=4$ and discard the terms $\mathcal{O}(D-4)$ and $\mathcal{O}\left(\mu^{-1}\right)$,

$$
\begin{aligned}
\rho_{q}^{\mathrm{UV}, 0}= & \frac{1}{16 \pi^{2} a^{4}}\left\{-\mu^{4}-(1-6 \xi) \mathcal{H}^{2} \mu^{2}-\frac{1}{2}(1-6 \xi)^{2}\left[-2 \mathcal{H}^{\prime \prime} \mathcal{H}+\left(\mathcal{H}^{\prime}\right)^{2}+3 \mathcal{H}^{4}\right] \frac{\mu^{D-4}}{D-4}\right. \\
& \left.+\frac{1}{2}\left[(1-6 \xi)^{2} \ln a-c\right]\left[-2 \mathcal{H}^{\prime \prime} \mathcal{H}+\left(\mathcal{H}^{\prime}\right)^{2}+3 \mathcal{H}^{4}\right]-\frac{3}{2}(1-6 \xi)^{2} \mathcal{H}^{4}\right\}, \\
p_{q}^{\mathrm{UV}, 0}= & \frac{1}{48 \pi^{2} a^{4}}\left\{-\mu^{4}+(1-6 \xi)\left(2 \mathcal{H}^{\prime}-\mathcal{H}^{2}\right) \mu^{2}-\frac{1}{2}\left[2 \mathcal{H}^{\prime \prime \prime}-2 \mathcal{H}^{\prime \prime} \mathcal{H}+\left(\mathcal{H}^{\prime}\right)^{2}-12 \mathcal{H}^{\prime} \mathcal{H}^{2}+3 \mathcal{H}^{4}\right] \frac{\mu^{D-4}}{D-4}\right. \\
+ & \frac{1}{2}\left[(1-6 \xi)^{2} \ln a-c\right]\left[2 \mathcal{H}^{\prime \prime \prime}-2 \mathcal{H}^{\prime \prime} \mathcal{H}+\left(\mathcal{H}^{\prime}\right)^{2}-12 \mathcal{H}^{\prime} \mathcal{H}^{2}+3 \mathcal{H}^{4}\right] \\
+ & \left.\frac{1}{6}(1-6 \xi)^{2}\left[2 \mathcal{H}^{\prime \prime \prime}-2 \mathcal{H}^{\prime \prime} \mathcal{H}+\left(\mathcal{H}^{\prime}\right)^{2}+24 \mathcal{H}^{\prime} \mathcal{H}^{2}-6 \mathcal{H}^{4}\right]\right\},
\end{aligned}
$$

where $c=(1-6 \xi)\left[\frac{1}{2}(1-6 \xi)\left(\gamma_{E}-\ln \pi\right)+2 \xi\right]$, and $\gamma_{E}$ is the Euler-Mascheroni constant.

The action for the counterterm needed to absorb the logarithmic divergence is

$$
S_{c t}=\alpha_{c t} S_{1}=\alpha_{c t} \int d^{D} x \sqrt{-g} R
$$

where

$$
\alpha_{c t}=\frac{1}{1152 \pi^{2}}\left[(1-6 \xi)^{2}\left(\frac{\mu^{D-4}}{D-4}\right)+\alpha_{f}\right]
$$

and $\alpha_{f}$ is a free finite constant (that can depend on $\mu$ logarithmically), to be determined by measurements in 
principle. $S_{c t}$ gives the following contribution to the energy-momentum tensor:

$$
\begin{aligned}
\alpha_{c t} \times{ }^{(1)} H_{\mu \nu} & =\alpha_{c t} \times \frac{(-2)}{\sqrt{-g}} \frac{\delta S_{1}}{\delta g^{\mu \nu}} \\
& =\alpha_{c t}\left(4 \nabla_{\mu} \nabla_{\nu} R-4 g_{\mu \nu} \square R+g_{\mu \nu} R^{2}-4 R_{\mu \nu} R\right) .
\end{aligned}
$$

For FLRW space-time ${ }^{(1)} H_{\mu \nu}$ is diagonal, and its expansion around $D=4$ reads

$$
\begin{aligned}
\rho_{q}^{c t}= & \frac{\alpha_{c t}}{a^{2}} \times{ }^{(1)} H_{00} \\
= & \frac{(1-6 \xi)^{2}}{32 \pi^{2} a^{4}}\left[-2 \mathcal{H}^{\prime \prime} \mathcal{H}+\left(\mathcal{H}^{\prime}\right)^{2}+3 \mathcal{H}^{4}\right]\left(\frac{\mu^{D-4}}{D-4}\right) \\
+ & \frac{(1-6 \xi)^{2}}{96 \pi^{2} a^{4}}\left[-4 \mathcal{H}^{\prime \prime} \mathcal{H}+2\left(\mathcal{H}^{\prime}\right)^{2}-6 \mathcal{H}^{\prime} \mathcal{H}^{2}+9 \mathcal{H}^{4}\right] \\
+ & \frac{\alpha_{f}}{32 \pi^{2} a^{4}}\left[-2 \mathcal{H}^{\prime \prime \prime} \mathcal{H}+\left(\mathcal{H}^{\prime}\right)^{2}+3 \mathcal{H}^{4}\right], \\
\delta_{i j} p_{q}^{c t}= & \frac{\alpha_{c t}}{a^{2}} \times{ }^{(1)} H_{i j} \\
= & \delta_{i j} \frac{(1-6 \xi)^{2}}{96 \pi^{2} a^{4}}\left[2 \mathcal{H}^{\prime \prime \prime}-2 \mathcal{H}^{\prime \prime} \mathcal{H}+\left(\mathcal{H}^{\prime}\right)^{2}\right. \\
& \left.-12 \mathcal{H}^{\prime} \mathcal{H}^{2}-3 \mathcal{H}^{4}\right]\left(\frac{\mu^{D-4}}{D-4}\right) \\
& +\delta_{i j} \frac{(1-6 \xi)^{2}}{288 \pi^{2} a^{4}}\left[2 \mathcal{H}^{\prime \prime \prime}+10 \mathcal{H}^{\prime \prime} \mathcal{H}+10\left(\mathcal{H}^{\prime}\right)^{2}\right. \\
& \left.-30 \mathcal{H}^{\prime} \mathcal{H}^{2}-3 \mathcal{H}^{4}\right]+\delta_{i j} \frac{\alpha_{f}}{96 \pi^{2} a^{4}}\left[2 \mathcal{H}^{\prime \prime \prime}\right. \\
& \left.-2 \mathcal{H}^{\prime \prime} \mathcal{H}+\left(\mathcal{H}^{\prime}\right)^{2}-12 \mathcal{H}^{\prime} \mathcal{H}^{2}-3 \mathcal{H}^{4}\right] .
\end{aligned}
$$

The terms divergent in $D=4$ in the two expressions above cancel the divergent terms in (A14) and (A15).

There is one more contribution to the energy-momentum tensor, which survives even in the conformal limit $\xi \rightarrow 1 / 6$, the so-called conformal anomaly [43,47,48]. It is not strictly necessary for renormalization on FLRW spacetime, but it is on more general ones, so we include it here. Its contribution on FLRW is

$$
\begin{aligned}
\rho_{q}^{c a}= & \frac{1}{2880 \pi^{2} a^{4}}\left[2 \mathcal{H}^{\prime \prime} \mathcal{H}-\left(\mathcal{H}^{\prime}\right)^{2}\right] \\
& +\frac{\alpha_{c a}}{32 \pi^{2} a^{4}}\left[2 \mathcal{H}^{\prime \prime} \mathcal{H}-\left(\mathcal{H}^{\prime}\right)^{2}-3 \mathcal{H}^{4}\right], \quad(\text { A21 }) \\
p_{q}^{c a}= & -\frac{1}{8640 \pi^{2} a^{4}}\left[2 \mathcal{H}^{\prime \prime \prime}-2 \mathcal{H}^{\prime \prime} \mathcal{H}+\left(\mathcal{H}^{\prime}\right)^{2}\right] \\
& +\frac{\alpha_{c a}}{96 \pi^{2} a^{4}}\left[-2 \mathcal{H}^{\prime \prime \prime}+2 \mathcal{H}^{\prime \prime} \mathcal{H}-\left(\mathcal{H}^{\prime}\right)^{2}+12 \mathcal{H}^{\prime} \mathcal{H}^{2}-3 \mathcal{H}^{4}\right],
\end{aligned}
$$

where $\alpha_{c a}$ is a free constant.
The final answer for the UV part of the energy-momentum tensor is obtained by adding all these three contributions together,

$$
\begin{aligned}
\rho_{q}^{\mathrm{UV}}= & \rho_{q}^{\mathrm{UV}, 0}+\rho_{q}^{c t}+\rho_{q}^{c a} \\
= & \frac{1}{16 \pi^{2} a^{4}}\left[-\mu^{4}-(1-6 \xi) \mathcal{H} \mu^{2}\right] \\
& +\frac{(1-6 \xi)^{2}}{32 \pi^{2} a^{4}}\left[-2 \mathcal{H}^{\prime \prime \prime} \mathcal{H}+\left(\mathcal{H}^{\prime}\right)^{2}+3 \mathcal{H}^{4}\right]\left(\ln a+\tilde{\alpha}_{f}\right) \\
& +\frac{(1-6 \xi)^{2}}{48 \pi^{2} a^{4}}\left[-2 \mathcal{H}^{\prime \prime} \mathcal{H}+\left(\mathcal{H}^{\prime}\right)^{2}-3 \mathcal{H}^{\prime} \mathcal{H}^{2}\right] \\
& +\frac{1}{2880 \pi^{2} a^{4}}\left[2 \mathcal{H}^{\prime \prime} \mathcal{H}-\left(\mathcal{H}^{\prime}\right)^{2}\right], \\
p_{q}^{\mathrm{UV}}= & p_{q}^{\mathrm{UV}, 0}+p_{q}^{c t}+p_{q}^{c a} \\
= & \frac{1}{48 \pi^{2} a^{4}}\left[\mu^{4}+(1-6 \xi)\left(2 \mathcal{H}^{\prime}-\mathcal{H}^{2}\right) \mu^{2}\right] \\
& +\frac{(1-6 \xi)^{2}}{96 \pi^{2} a^{4}}\left[2 \mathcal{H}^{\prime \prime \prime}-2 \mathcal{H}^{\prime \prime} \mathcal{H}+\left(\mathcal{H}^{\prime}\right)^{2}\right. \\
& \left.-12 \mathcal{H}^{\prime} \mathcal{H}^{2}+3 \mathcal{H}^{4}\right]\left(\ln a+\tilde{\alpha}_{f}\right) \\
& +\frac{(1-6 \xi)^{2}}{288 \pi^{2} a^{4}}\left[4 \mathcal{H}^{\prime \prime \prime}+8 \mathcal{H}^{\prime \prime} \mathcal{H}+11\left(\mathcal{H}^{\prime}\right)^{2}-6 \mathcal{H}^{\prime} \mathcal{H}^{2}-9 \mathcal{H}^{4}\right] \\
& -\frac{1}{8640 \pi^{2} a^{4}}\left[2 \mathcal{H}^{\prime \prime \prime}-2 \mathcal{H}^{\prime \prime} \mathcal{H}+\left(\mathcal{H}^{\prime}\right)^{2}\right],
\end{aligned}
$$

where all the free constants $c, \alpha_{f}$ and $\alpha_{c a}$ have been collected in $\tilde{\alpha}_{f}$ since they multiply the same contributions. Energy density and pressure given above satisfy the conservation equation (5) by themselves. Specializing to FLRW backgrounds with constant $\epsilon$ parameter, the UV contributions to the energy density and pressure are

$$
\begin{aligned}
\rho_{q}^{\mathrm{UV}}= & \frac{1}{16 \pi^{2} a^{4}}\left\{-\mu^{4}-(1-6 \xi) \mathcal{H}^{2} \mu^{2}\right. \\
& +\frac{3}{2}(1-6 \xi)^{2} \epsilon(2-\epsilon) \mathcal{H}^{4}(\ln a+\tilde{\alpha}) \\
& \left.-(1-6 \xi)^{2}(1-\epsilon)(2-\epsilon) \mathcal{H}^{4}+\frac{1}{60}(1-\epsilon)^{2} \mathcal{H}^{4}\right\}, \\
p_{q}^{\mathrm{UV}}= & \frac{1}{48 \pi^{2} a^{4}}\left\{-\mu^{4}+(1-6 \xi)(1-2 \epsilon) \mathcal{H}^{2} \mu^{2}\right. \\
& -\frac{3}{2}(1-6 \xi)^{2} \epsilon(2-\epsilon)(3-4 \epsilon) \mathcal{H}^{4}(\ln a+\tilde{\alpha}) \\
& +\frac{1}{2}(1-6 \xi)^{2}(2-\epsilon)\left(6-17 \epsilon+8 \epsilon^{2}\right) \mathcal{H}^{4} \\
& \left.-\frac{1}{60}(1-\epsilon)^{2}(3-4 \epsilon) \mathcal{H}^{4}\right\} .
\end{aligned}
$$

The full answer for the one-loop expectation value for energy density and pressure is obtained by adding up the UV and IR contributions and taking the limit $\mu \rightarrow \infty$, 


$$
\rho_{q}=\lim _{\mu \rightarrow \infty}\left(\rho_{q}^{\mathrm{UV}}+\rho_{q}^{\mathrm{IR}}\right), \quad p_{q}=\lim _{\mu \rightarrow \infty}\left(p_{q}^{\mathrm{UV}}+p_{q}^{\mathrm{IR}}\right) .
$$

\section{APPENDIX B: COSMOLOGICAL PARAMETERS}

This appendix serves as a reminder of how to relate quantities $\mathcal{H}_{i}$ we have used in the calculation with the values of cosmological parameters usually used. We basically use relation (8),

$$
\mathcal{H}_{i}=\mathcal{H}_{i-1}\left(\frac{a_{i}}{a_{i-1}}\right)^{1-\epsilon_{i}}=\mathcal{H}_{i-1} e^{\left(1-\epsilon_{i}\right) N_{i}},
$$

to accomplish this, where $N_{i}=\ln \left(a_{i} / a_{i-1}\right)$ is the number of $e$-foldings of the $i$ th period. The cosmological parameters (except $\epsilon_{I}$ ) are taken from [3]. By taking the Hubble rate today (with $a_{\text {tod }}=1$ ) to be

$$
H_{\text {tod }}=68 \frac{\mathrm{km}}{\mathrm{Mpc} \mathrm{s}}=\mathcal{H}_{\text {tod }},
$$

and the redshift of radiation-matter equality,

$$
z_{\mathrm{eq}}=3270=\frac{1}{a_{2}}+1
$$

we get that the (conformal) Hubble rate at the time of transition between radiation and matter period is

$$
\mathcal{H}_{2}=3888 \frac{\mathrm{km}}{\mathrm{Mpc} \mathrm{s}},
$$

and the duration of matter period expressed in the number of $e$-foldings

$$
N_{M}=8.09 \text {. }
$$

The condition of minimal inflation that the conformal Hubble rate at the beginning of inflation be equal to the one today, $\mathcal{H}_{0, \min }=\mathcal{H}$, gives one condition on the $e$-foldings,

$$
0=\left(1-\epsilon_{I}\right) N_{I, \min }-N_{R}-\frac{1}{2} N_{M} .
$$

The other condition needed is supplied by the amplitude of the scalar perturbations in the CMB. It fixes the physical Hubble rate

$$
H_{*}=3.45 \times 10^{13} \frac{\mathrm{GeV}}{\hbar}=1.618 \times 10^{60} \frac{\mathrm{m}}{\mathrm{Mpc} \mathrm{s}}
$$

at the time the mode at the pivotal scale $k_{*}=0.002 \mathrm{Mpc}^{-1}$ left the horizon during inflation. For minimal inflation the Hubble scale is the same as today, $k_{0}=0.00026 \mathrm{Mpc}^{-1}$, which gives us

$$
\frac{k_{*}}{k_{0}}=\frac{\mathcal{H}_{*}}{\mathcal{H}_{0, \text { min }}}=\left(\frac{a_{*}}{a_{0, \text { min }}}\right)^{1-\epsilon_{I}} \text {. }
$$

Using the minimal inflation assumption $H_{\text {tod }}=\mathcal{H}_{0}$, this condition can be rewritten as

$$
N_{I, \text { min }}+N_{R}+N_{R}=\ln \left[\frac{H_{*}}{H_{\mathrm{tod}}}\left(\frac{k_{0}}{k_{*}}\right)^{\frac{\epsilon_{I}}{1-\varepsilon_{I}}}\right] .
$$

Results from [3] would suggest that $\epsilon_{I}=0.01$, which, together with the two conditions (B6) and (B9) fixes the duration of radiation period and minimal inflation,

$$
N_{R}=60.77, \quad N_{I, \text { min }}=65.47,
$$

from where we can infer the Hubble rate at the end of inflation (at the beginning of inflation it is the same as today)

$$
\mathcal{H}_{1}=9.59 \times 10^{29} \frac{\mathrm{km}}{\mathrm{Mpc} \mathrm{s}} .
$$

\section{APPENDIX C: BACKREACTION DURING RADIATION PERIOD}

In this appendix we repeat the calculation from Sec. VIII, but this time for the backreaction during radiation period (Fig. 1). The hierarchy of scales assumed is $\mathcal{H}_{0} \ll \mathcal{H} \ll \mathcal{H}_{1}$. This is strictly valid only some time after the transition from inflation to radiation period when the conformal Hubble rate has dropped enough.

There is no need to repeat here step by step all the approximation steps for Bogolyubov coefficients from Sec. IX. The approximation for $Z_{\mathrm{Bog}}$. here is obtained by making substitutions $A_{3,1} \rightarrow A_{2,1}, \nu_{I} \rightarrow \nu_{R}=\frac{1}{2}$, and $u_{M} \rightarrow u_{R}$ in the expression (86),

$$
\begin{aligned}
Z_{\mathrm{Bog}}(k, \eta)= & \frac{8\left|A_{2,1}\right|^{2}}{k^{2+2 \nu_{I}}}\left[\frac{1}{2} \frac{\partial^{2}}{\partial \eta_{0}^{2}}+2 k^{2}+f_{I}\left(\eta_{0}\right)\right] \\
& \times \Re^{2}\left[u_{I}\left(k, \eta_{0}\right)\right] \Re^{2}\left[u_{R}(k, \eta)\right],
\end{aligned}
$$

where

$$
\left|A_{2,1}\right|^{2}=\frac{\Gamma^{2}\left(\nu_{I}\right)\left(\nu_{I}-\frac{1}{2}\right)^{2}}{16 \pi}\left[2\left(1-\epsilon_{I}\right) \mathcal{H}_{1}\right]^{2 \nu_{I}+1} .
$$

The integrals (88) for $J_{s}$ to be evaluated here are

$$
\begin{aligned}
\mathcal{J}_{s} & =\int_{0}^{\mu} d k k^{2 s-1-2 \nu_{I}-2 \nu_{M}} \Re^{2}\left[u_{I}\left(k, \eta_{0}\right)\right] \Re^{2}\left[u_{R}(k, \eta)\right] \\
& =\frac{\pi}{8\left(1-\epsilon_{I}\right) \mathcal{H}_{0}} \int_{0}^{\mu} d k k^{2 s-3-2 \nu_{I}} J_{\nu_{I}}^{2}\left(\frac{k}{\left(1-\epsilon_{I}\right) \mathcal{H}_{0}}\right) \sin ^{2}\left(\frac{k}{\mathcal{H}}\right), \\
& s=1,2,3,
\end{aligned}
$$


and the solution for them is ( $\mu$-independent term in the $1 / \mu$ expansion)

$$
\begin{aligned}
\mathcal{J}_{s}= & \frac{\sqrt{\pi} \Gamma(s-1) \Gamma\left(\frac{3}{2}-s+\nu_{I}\right)}{32 \Gamma\left(2-s+\nu_{I}\right) \Gamma\left(2-s+2 \nu_{I}\right)}\left[\left(1-\epsilon_{I}\right) \mathcal{H}_{0}\right]^{-3+2 s-2 \nu_{I}} \\
& \times\left[1-{ }_{3} F_{2}\left(-1+s,-1+s-2 \nu_{I},-1+s-\nu_{I} ; \frac{1}{2},-\frac{1}{2}+s-\nu_{I} ; \frac{\left(1-\epsilon_{I}\right)^{2} \mathcal{H}_{0}^{2}}{\mathcal{H}^{2}}\right)\right] \\
& +2^{-1-2 s+2 \nu_{I}} \Gamma\left(-3+2 s-2 \nu_{I}\right) \sin \left[\pi\left(s-\nu_{I}\right)\right] \mathcal{H}^{-3+2 s-2 \nu_{I}} \\
& \times{ }_{3} F_{2}\left(\frac{1}{2}, \frac{1}{2}-\nu_{I}, \frac{1}{2}+\nu_{I} ; 2-s+\nu_{I}, \frac{5}{2}-s+\nu_{I} ; \frac{\left(1-\epsilon_{I}\right)^{2} \mathcal{H}_{0}^{2}}{\mathcal{H}^{2}}\right) .
\end{aligned}
$$

This can be further simplified by recognizing that during radiation period $\mathcal{H} \gg \mathcal{H}_{0}$ is always satisfied, so we can expand in $\mathcal{H}_{0} / \mathcal{H}$,

$$
\begin{aligned}
\mathcal{J}_{s} \approx & 2^{-1-2 s+2 \nu_{I}} \Gamma\left(-3+2 s-2 \nu_{I}\right) \sin \left[\pi\left(s-\nu_{I}\right)\right] \mathcal{H}^{-3+2 s-2 \nu_{I}} \\
& +\frac{\sqrt{\pi} \Gamma(s) \Gamma\left(\frac{1}{2}-s+\nu_{I}\right)}{16 \Gamma\left(1-s+\nu_{I}\right) \Gamma\left(1-s+2 \nu_{I}\right)} \frac{\left[\left(1-\epsilon_{I}\right) \mathcal{H}_{0}\right]^{-1+2 s-2 \nu_{I}}}{\mathcal{H}^{2}},
\end{aligned}
$$

where, depending on $s$ and $\nu_{I}$ the first or the second term might be dominant. Now we can form $\mathcal{I}_{s}$ integrals easily via (92), and from (42) and (43) calculate the energy density and pressure, which to leading order in $\mathcal{H}_{0} / \mathcal{H}$ are

$$
\begin{aligned}
& \rho_{q}=\frac{3 \mathcal{H}_{0}^{4}}{16 \pi^{2} a^{4}}\left(\frac{\mathcal{H}_{1}}{\mathcal{H}_{0}}\right)^{2 \nu_{I}+1} \frac{\xi(1-6 \xi)\left(2-\epsilon_{I}\right)\left(1-\epsilon_{I}\right)^{2}\left(\nu_{I}-\frac{1}{2}\right)^{2}}{\left(\nu_{I}-\frac{3}{2}\right)}, \\
& p_{q}=\frac{\mathcal{H}_{0}^{4}}{16 \pi^{2} a^{4}}\left(\frac{\mathcal{H}_{1}}{\mathcal{H}_{0}}\right)^{2 \nu_{I}+1} \frac{\xi(1-6 \xi)\left(2-\epsilon_{I}\right)\left(1-\epsilon_{I}\right)^{2}\left(\nu_{I}-\frac{1}{2}\right)^{2}}{\left(\nu_{I}-\frac{3}{2}\right)} .
\end{aligned}
$$

The backreaction during radiation period behaves as an ideal fluid with a constant equation of state parameter

$$
w_{q}=\frac{1}{3}=w_{b},
$$

which means it scales just like radiation that dominates the background. Therefore, the ratio of the quantum to classical energy density during radiation period is constant, and for the choice of parameters of interest from Sec. IX, $N_{I}=69$ and $\xi=-0.051614$, this ratio is

$$
\frac{\rho_{q}}{\rho_{b}}=-0.4335
$$

Therefore, the dominant contribution to the energy density is negative, scales the same as the background, but is smaller than the background.

This result does not reproduce the $\epsilon_{I} \rightarrow 0, \xi \rightarrow 0, \mathcal{H}_{0} \rightarrow 0$ limit of [1]. The reason is that in this limit some $\mu$-dependent terms become $\mu$-independent, and of the same order as the leading term. Fortunately, there is another way we can calculate this result, by evaluating simpler integrals, and we can reproduce the limit of [1].

We go back to integrals $\mathcal{I}_{s}$ in (48) and split the integration intervals by introducing another scale $\mu_{0}$ such that $\mathcal{H}_{0} \ll \mu_{0} \ll \mathcal{H} \ll \mu \ll \mathcal{H}_{1}$,

$$
\mathcal{I}_{s}^{\left(0, \mu_{0}\right)}+\mathcal{I}_{s}^{\left(\mu_{0}, \mu\right)}=\left(\int_{0}^{\mu_{0}}+\int_{\mu_{0}}^{\mu}\right) d k k^{2 s} Z_{\mathrm{Bog}}(k, \eta)
$$

On the interval $\left(0, \mu_{0}\right)$ we can follow the same procedure (C1)-(C3), just that the integration is up to $\mu_{0}$, and we can further expand integral $(\mathrm{C} 3)$ in $k / \mathcal{H}$ to get 


$$
\begin{aligned}
\mathcal{J}_{s}^{\left(0, \mu_{0}\right)} & =\frac{\pi}{8\left(1-\epsilon_{I}\right) \mathcal{H}_{0} \mathcal{H}^{2}} \int_{0}^{\mu_{0}} d k k^{2 s-1-2 \nu_{I}} J_{\nu_{I}}^{2}\left(\frac{k}{\left(1-\epsilon_{I}\right) \mathcal{H}_{0}}\right) \\
& =\frac{2^{-4-2 \nu_{I}} \pi \mu_{0}^{2 s}\left[\left(1-\epsilon_{I}\right) \mathcal{H}_{0}\right]^{-1-2 \nu_{I}}}{s \Gamma^{2}\left(1+\nu_{I}\right) \mathcal{H}^{2}} F_{3}\left(s, \frac{1}{2}+\nu_{I} ; 1+s, 1+\nu_{I}, 1+2 \nu_{I} ; \frac{-\mu_{0}^{2}}{\left(1-\epsilon_{I}\right)^{2} \mathcal{H}_{0}^{2}}\right) \\
& =\frac{\sqrt{\pi} \Gamma(s) \Gamma\left(\frac{1}{2}-2+\nu_{I}\right)\left[\left(1-\epsilon_{I}\right) \mathcal{H}_{0}\right]^{-1+2 s-2 \nu_{I}}}{16 \Gamma\left(1-s+\nu_{I}\right) \Gamma\left(1-s+2 \nu_{I}\right) \mathcal{H}^{2}}+\frac{\mu_{0}^{-1+2 s-2 \nu_{I}}}{16 \mathcal{H}^{2}\left(-\frac{1}{2}+s-\nu_{I}\right)},
\end{aligned}
$$

where we were careful to write down the leading order $\mu_{0}$-dependent term. The contribution to energy density from $\left(0, \mu_{0}\right)$ interval is then

$$
\begin{aligned}
\rho_{q}^{\left(0, \mu_{0}\right)}= & \frac{3 \mathcal{H}_{0}^{4}}{16 \pi^{2} a^{4}}\left(\frac{\mathcal{H}_{1}}{\mathcal{H}_{0}}\right)^{2 \nu_{I}+1} \frac{\xi(1-6 \xi)\left(2-\epsilon_{I}\right)\left(1-\epsilon_{I}\right)^{2}\left(\nu_{I}-\frac{1}{2}\right)^{2}}{\left(\nu_{I}-\frac{3}{2}\right)} \\
& -\frac{\mu_{0}^{3-2 \nu_{I}}}{\left(\nu_{I}-\frac{3}{2}\right)} \times \frac{6 \xi \Gamma^{2}\left(\nu_{I}\right)\left(\nu_{I}-\frac{1}{2}\right)^{2}}{32 \pi^{3}}\left[2\left(1-\epsilon_{I}\right) \mathcal{H}_{1}\right]^{2 \nu_{I}+1},
\end{aligned}
$$

where we have kept the term that is $\mu_{0}$-independent in the $\nu_{I} \rightarrow 3 / 2$ limit. This contribution contributes the dominant term for $\xi<0$, and vanishes in the $\xi \rightarrow 0, \epsilon_{I} \rightarrow 0, \mathcal{H}_{0} \rightarrow 0$ limit.

On the interval $\left(\mu_{0}, \mu\right)$ we can again expand the Bogolyubov coefficients in $k / \mathcal{H}_{1}$, which amounts to the integrand being approximated by

$$
Z_{\mathrm{Bog}}=\frac{4\left|A_{2,1}\right|^{2}}{k^{2 \nu_{I}+1}}\left|\alpha_{1,0}-\beta_{1,0}\right|^{2} \Re^{2}\left[u_{R}(k, \eta)\right] .
$$

From (83) we have that

$\left|\alpha_{1,0}-\beta_{1,0}\right|^{2}=\frac{2}{k}\left[\Re^{2}\left[u_{I}^{\prime}\left(k, \eta_{0}\right)\right]+k^{2} \Re^{2}\left[u_{I}\left(k, \eta_{0}\right)\right]\right]$,

which can be expanded in $\mathcal{H}_{0} / k$ on this interval,

$$
\left|\alpha_{1,0}-\beta_{1,0}\right|^{2}=1 \text {. }
$$

Then we have for the $\mathcal{I}_{s}$ integrals (expanded in $\mu$ and $\mu_{0}$ )

$$
\begin{aligned}
\mathcal{I}_{s}^{\left(\mu_{0}, \mu\right)}= & 2\left|A_{2,1}\right|^{2} \int_{\mu_{0}}^{\mu} d k k^{2 s-2-2 \nu_{I}} \sin ^{2}\left(\frac{k}{\mathcal{H}}\right) \\
= & 2\left|A_{2,1}\right|^{2}\left[-4^{-s+\nu_{I}} \Gamma\left(-1+2 s-2 \nu_{I}\right) \sin \left[\pi\left(s-\nu_{I}\right)\right]\right. \\
& \left.+\frac{\mu^{-1+2 s-2 \nu_{I}}}{2\left(-1+2 s-2 \nu_{I}\right)}\right] .
\end{aligned}
$$

Away from the limit in [1] this interval gives a subdominant contribution to the energy density. Therefore, here we present just that particular limit, which turns out to be

$$
\rho_{q}^{\left(\mu_{0}, \mu\right)}=\frac{\mathcal{H}_{1}^{4}}{8 \pi^{2} a^{4}} \ln (a)
$$

which is exactly the one from [1]. Actually, one has to go through similar difficulties in the cases where $\nu_{I}$ or $\nu_{M}$ happen to have a half-integer value.

\section{APPENDIX D: LIMITS $\mathcal{H} \gg \mathcal{H}_{0}$ AND $\mathcal{H} \ll \mathcal{H}_{0}$ OF THE RESULT DURING MATTER ERA}

In this appendix we present the direct computation of the limits $\mathcal{H} \gg \mathcal{H}_{0}$ (early matter period) and $\mathcal{H} \ll \mathcal{H}_{0}$ (very late matter period). Even though the former physically makes sense in the limited range of nonminimal coupling, and the latter not at all, it is still useful as an independent check of the results presented in Sec. IX. Instead of calculating the $\mathcal{J}_{s}$ integrals, we calculate the $\mathcal{I}_{s}$ integrals (48) straight away.

\section{Limit $\mathcal{H} \ll \mathcal{H}_{0}$}

In this case we solve the $\mathcal{I}_{s}$ integrals (48) with the hierarchy $\mathcal{H} \ll \mu \ll \mathcal{H}_{0} \ll \mathcal{H}_{2} \ll \mathcal{H}_{1}$. Here we can use the approximation of Sect. VII B for the entire Bogolyubov coefficients, namely

$$
\alpha_{3,0} \approx \beta_{3,0} \approx \frac{i A_{3,0}}{k^{1 / 2+\nu_{M}}} \approx-i \beta_{3,0}^{*},
$$

where

$$
\begin{aligned}
A_{3,0}= & \frac{\Gamma\left(\nu_{M}\right)\left(2-\epsilon_{I}\right)}{8 \sqrt{\pi} \nu_{I}\left(1-\epsilon_{I}\right)}(1-6 \xi)\left(\nu_{M}+\frac{3}{2}\right) \\
& \times \mathcal{H}_{0}^{-\nu_{I}+1 / 2} \mathcal{H}_{1}^{\nu_{I}+1 / 2} \mathcal{H}_{2}^{\nu_{M}-1 / 2} .
\end{aligned}
$$

This allows us to write the $\mathcal{I}_{s}$ integrals as

$$
\begin{aligned}
\mathcal{I}_{s} & =4\left|A_{3,0}\right|^{2} \int_{0}^{\mu} d k k^{2 s-1-2 \nu_{M}} \Re^{2}\left[u_{M}(k, \eta)\right] \\
& =\frac{2 \pi\left|A_{3,0}\right|^{2}}{\mathcal{H}} \int_{0}^{\mu} d k k^{2 s-1-2 \nu_{M}} J_{\nu_{M}}^{2}\left(\frac{2 k}{\mathcal{H}}\right),
\end{aligned}
$$


which evaluates to

$$
\begin{aligned}
\mathcal{I}_{s}= & \left|A_{3,0}\right|^{2} \frac{\pi \mu^{2 s} \mathcal{H}^{-1-2 \nu_{M}}}{s \Gamma^{2}\left(1+\nu_{M}\right)}{ }_{2} F_{3} \\
& \times\left(s, \frac{1}{2}+\nu_{M} ; 1+s, 1+\nu_{M}, 1+2 \nu_{M} ;-\frac{4 \mu^{2}}{\mathcal{H}^{2}}\right) \\
= & \left|A_{3,0}\right|^{2} \frac{2^{-2 s+2 \nu_{M}} \sqrt{\pi} \Gamma(s) \Gamma\left(\frac{1}{2}-s+\nu_{M}\right)}{\Gamma\left(1-s+\nu_{M}\right) \Gamma\left(1-s+2 \nu_{M}\right)} \mathcal{H}^{-1+2 s-2 \nu_{M}} .
\end{aligned}
$$

Plugging this into (93) and (94) gives the very late-time limit for the energy density and pressure,

$$
\begin{aligned}
\rho_{q}= & -\frac{(1-6 \xi)^{3}\left(2-\epsilon_{I}\right)^{2}\left(\nu_{M}+\frac{3}{2}\right)^{2}\left(\nu_{M}-1\right)\left(\nu_{M}-\frac{5}{2}\right)}{2^{9} \pi^{2}\left(1-\epsilon_{I}\right)^{2} \nu_{I}^{2}\left(\nu_{M}-\frac{1}{2}\right)\left(\nu_{M}-\frac{3}{2}\right)} \\
& \times \mathcal{H}_{0}^{-2 \nu_{I}+1} \mathcal{H}^{2 \nu_{I}+1} \mathcal{H}_{2}^{2 \nu_{M}-1} \frac{\mathcal{H}^{3-2 \nu_{M}}}{a^{4}}, \\
p_{q}= & -\frac{(1-6 \xi)^{3}\left(2-\epsilon_{I}\right)^{2}\left(\nu_{M}+\frac{3}{2}\right)^{2}\left(\nu_{M}-1\right)\left(\nu_{M}-\frac{5}{2}\right)}{2^{9} \pi^{2}\left(1-\epsilon_{I}\right)^{2} \nu_{I}^{2}\left(\nu_{M}-\frac{1}{2}\right)\left(\nu_{M}-\frac{3}{2}\right)} \\
& \times \frac{\left(\nu_{M}-\frac{5}{2}\right)}{3} \times \mathcal{H}_{0}^{-2 \nu_{I}+1} \mathcal{H}^{2 \nu_{I}+1} \mathcal{H}_{2}^{2 \nu_{M}-1} \frac{\mathcal{H}^{3-2 \nu_{M}}}{a^{4}} .
\end{aligned}
$$

This is precisely the limit one gets from expanding (90) in $\mathcal{H} / \mathcal{H}_{0} \ll 1$ for and then using it to calculate the energy density and pressure. In this limit the backreaction behaves as a fluid with a constant equation of state,

$$
w_{q}=\frac{1}{3}\left(\nu_{M}-\frac{5}{2}\right),
$$

and the sign of the energy density is determined by the sign of $-w_{q}$. The potentially interesting case where $\nu_{M}>5 / 2$ and the backreaction grows with respect to the background fluid corresponds to $\xi<-1 / 3$ which is far out of the region where the backreaction stays small during the evolution of the Universe. In fact, it already becomes dominant in inflation. In the end, this limit does not tell us much other than provides a check for the calculation in Secs. VIII and IX.

Generally, the late-time scaling in the limit $\mathcal{H} \ll \mathcal{H}_{i}$, where $\mathcal{H}_{i}$ refer to the Hubble rates at any number $n$ of transitions will depend only on the starting and ending deceleration periods as $\sim a^{-4} \mathcal{H}^{4-2 \nu_{0}-2 \nu_{n}}$. That follows from the IR leading order term in the Bogolyubov coefficients derived in Sec. VII B where it was found that $\alpha \approx \beta \approx i A_{n, 0} k^{-\nu_{0}-\nu_{n}}$. The amplitude, of course, depends on the number and type of transitions.

\section{Limit $\mathcal{H} \gg \mathcal{H}_{0}$}

This limit corresponds to the early matter era (up until today for minimal inflation). In this limit we can use approximation (82) for the integrand of $\mathcal{I}_{s}$ in (48),

$\mathcal{I}_{s}^{(0, \mu)}=4\left|A_{3,1}\right|^{2} \int_{0}^{\mu_{0}} d k k^{2 s-2 \nu_{I}-2 \nu_{M}}\left|\alpha_{1,0}-\beta_{1,0}\right|^{2} \Re^{2}\left[u_{M}(k, \eta)\right]$,

where

$$
\left|\alpha_{1,0}-\beta_{1,0}\right|^{2}=\frac{2}{k}\left[\Re^{2}\left[u_{I}^{\prime}\left(k, \eta_{0}\right)\right]+k^{2} \Re\left[u_{I}\left(k, \eta_{0}\right)\right]\right] .
$$

Furthermore, we split the integration by another scale $\mu_{0}$, where the hierarchy is

$$
\mathcal{H}_{0} \ll \mu_{0} \ll \mathcal{H} \ll \mu \ll \mathcal{H}_{2} \ll \mathcal{H}_{1} .
$$

In the first of the two integrals we can expand in $k / \mathcal{H}$. Performing this expansion and using (84) leads to

$$
\mathcal{I}_{s}^{\left(0, \mu_{0}\right)}=8\left|A_{3,1}\right|^{2}\left\{2 \mathcal{J}_{s+1}^{\left(0, \mu_{0}\right)}+\left[\frac{1}{2} \frac{\partial^{2}}{\partial \eta_{0}^{2}}+f_{I}\left(\eta_{0}\right)\right] \mathcal{J}_{s}^{\left(0, \mu_{0}\right)}\right\},
$$

where

$$
\begin{aligned}
\mathcal{J}_{s}^{\left(0, \mu_{0}\right)} & =\frac{\pi^{2} \mathcal{H}^{-1-2 \nu_{M}}}{8\left(1-\epsilon_{I}\right) \Gamma^{2}\left(1+\nu_{M}\right) \mathcal{H}_{0}} \int_{0}^{\mu_{0}} d k k^{2 s-1-2 \nu_{I}} J_{\nu_{I}}^{2}\left(\frac{k}{\left(1-\epsilon_{I}\right) \mathcal{H}_{0}}\right) \\
& =\frac{2^{-4-2 \nu_{I}} \pi^{2} \mathcal{H}^{-1-2 \nu_{M}} \mu_{0}^{2 s}\left[\left(1-\epsilon_{I}\right) \mathcal{H}_{0}\right]^{-1-2 \nu_{I}}}{s \Gamma^{2}\left(1+\nu_{I}\right) \Gamma^{2}\left(1+\nu_{M}\right)} \times{ }_{2} F_{3}\left(2, \frac{1}{2}+\nu_{I} ; 1+s, 1+\nu_{I}, 1+2 \nu_{I} ; \frac{-\mu_{0}^{2}}{\left(1-\epsilon_{I}\right)^{2} \mathcal{H}_{0}^{2}}\right) \\
& =\frac{\pi^{3 / 2} \Gamma(s) \Gamma\left(\frac{1}{2}-s+\nu_{I}\right)}{16 \Gamma\left(1-s+\nu_{I}\right) \Gamma\left(1-s+2 \nu_{I}\right) \Gamma^{2}\left(1+\nu_{M}\right)}\left[\left(1-\epsilon_{I}\right) \mathcal{H}_{0}\right]^{-1+2 s-2 \nu_{I}} \mathcal{H}^{-1-2 \nu_{I}}
\end{aligned}
$$

This is precisely the leading order term in the limit $\mathcal{H} \gg \mathcal{H}_{0}$ of the full result (89) so here we must reproduce the same limit for energy density and pressure. So we have for the $\mu_{0}$-independent contribution to the $\mathcal{I}_{s}$ integrals 


$$
\mathcal{I}_{s}^{\left(0, \mu_{0}\right)}=8\left|A_{3,1}\right|^{2} \frac{2^{-3+2 s-2 \nu_{I}} \pi^{2}(1-6 \xi)\left(2-\epsilon_{I}\right)\left(2 \nu_{I}+1-2 s\right) \Gamma(s) \Gamma\left(-1+2 \nu_{I}-2 s\right)}{\left(1-\epsilon_{I}\right)^{2} \Gamma\left(\nu_{I}-s\right) \Gamma\left(1-s+\nu_{I}\right) \Gamma\left(1-s+2 \nu_{I}\right) \Gamma^{2}\left(1+\nu_{M}\right)} \times\left[\left(1-\epsilon_{I}\right) \mathcal{H}_{0}\right]^{1+2 s-2 \nu_{I}} \mathcal{H}^{-1-2 \nu_{I}} .
$$

On the other interval $\left(\mu_{0}, \mu\right)$ we can expand in $\mathcal{H}_{0} / k$, where

$$
\left|\alpha_{1,0}-\beta_{1,0}\right|^{2}=1
$$

to leading order. This leads to

$$
\mathcal{I}_{s}^{\left(\mu_{0}, \mu\right)}=\frac{2 \pi\left|A_{3,1}\right|^{2}}{\mathcal{H}} \int_{\mu_{0}}^{\mu} d k k^{2 s-2 \nu_{I}-2 \nu_{M}} J_{\mu_{M}}^{2}\left(\frac{2 k}{\mathcal{H}}\right)=\left|A_{3,1}\right|^{2} \frac{2^{-1-2 s+2 \nu_{I}+2 \nu_{M}} \sqrt{\pi} \Gamma\left(\frac{1}{2}+s-\nu_{I}\right) \Gamma\left(-s+\nu_{I}+\nu_{M}\right)}{\Gamma\left(\frac{1}{2}-s+\nu_{I}+\nu_{M}\right) \Gamma\left(\frac{1}{2}-s+\nu_{I}+2 \nu_{M}\right)} \mathcal{H}^{2 s-2 \nu_{I}-2 \nu_{M}},
$$

to leading order for the $\mu$-independent terms. This contribution is suppressed compared to (D13). Therefore, the leading contribution to energy density and pressure during early matter era is

$$
\begin{aligned}
& \rho_{q}=-\frac{(1-6 \xi)\left(1-\epsilon_{I}\right)^{2}\left(2-\epsilon_{I}\right)\left(\nu_{I}-\frac{1}{2}\right)^{2}\left(\nu_{M}+\frac{3}{2}\right)^{2}\left(\nu_{M}-\frac{3}{2}\right)\left(\nu_{M}+\frac{1}{2}\right)}{2^{8} \pi^{2} \nu_{M}\left(\nu_{I}-\frac{3}{2}\right)} \mathcal{H}_{0}^{3-2 \nu_{I}} \mathcal{H}_{1}^{1+2 \nu_{I}} \mathcal{H}_{2}^{-1+2 \nu_{M}} \mathcal{H}^{1-2 \nu_{M}} a^{-4} \\
& p_{q}=-\frac{(1-6 \xi)\left(1-\epsilon_{I}\right)^{2}\left(2-\epsilon_{I}\right)\left(\nu_{I}-\frac{1}{2}\right)^{2}\left(\nu_{M}+\frac{3}{2}\right)^{2}\left(\nu_{M}-\frac{3}{2}\right)\left(\nu_{M}+\frac{1}{2}\right)}{2^{8} \pi^{2} \nu_{M}\left(\nu_{I}-\frac{3}{2}\right)} \times \frac{\left(\frac{3}{2}-\nu_{M}\right)}{3} \mathcal{H}_{0}^{3-2 \nu_{I}} \mathcal{H}_{1}^{1+2 \nu_{I}} \mathcal{H}_{2}^{-1+2 \nu_{M}} \mathcal{H}^{1-2 \nu_{M}} a^{-4} .
\end{aligned}
$$

This result corresponds to the main result found in [1] for the limit $\epsilon_{I} \rightarrow 0, \xi \rightarrow 0, \mathcal{H}_{0} \rightarrow 0$.

[1] D. Glavan, T. Prokopec, and V. Prymidis, Phys. Rev. D 89, 024024 (2014).

[2] H. Aoki, S. Iso, and Y. Sekino, Phys. Rev. D 89, 103536 (2014).

[3] P. Ade et al. (BICEP2 Collaboration), Phys. Rev. Lett. 112, 241101 (2014).

[4] E. W. Kolb, S. Matarrese, and A. Riotto, New J. Phys. 8, 322 (2006).

[5] E. Barausse, S. Matarrese, and A. Riotto, Phys. Rev. D 71, 063537 (2005).

[6] C. M. Hirata and U. Seljak, Phys. Rev. D 72, 083501 (2005).

[7] C. Ringeval, T. Suyama, T. Takahashi, M. Yamaguchi, and S. Yokoyama, Phys. Rev. Lett. 105, 121301 (2010).

[8] T. Janssen and T. Prokopec, Phys. Rev. D 83, 084035 (2011).

[9] L. Abramo and R. Woodard, Phys. Rev. D 65, 063515 (2002).

[10] L. Abramo and R. Woodard, Phys. Rev. D 65, 043507 (2002).

[11] L. Abramo and R. Woodard, Phys. Rev. D 60, 044011 (1999).

[12] L. Abramo and R. Woodard, Phys. Rev. D 60, 044010 (1999).

[13] G. Geshnizjani and R. Brandenberger, Phys. Rev. D 66, 123507 (2002).
[14] G. Geshnizjani and R. Brandenberger, J. Cosmol. Astropart. Phys. 04 (2005) 006.

[15] L. Abramo and R. Woodard, Phys. Rev. D 65, 063516 (2002).

[16] T. Janssen and T. Prokopec, Ann. Phys. (Amsterdam) 325, 948 (2010).

[17] G. Marozzi and G. P. Vacca, Phys. Rev. D 88, 027302 (2013).

[18] G. Marozzi and G. P. Vacca, Classical Quantum Gravity 29, 115007 (2012).

[19] G. Marozzi, G.P. Vacca, and R. H. Brandenberger, J. Cosmol. Astropart. Phys. 02 (2013) 027.

[20] T. Prokopec, N. Tsamis, and R. Woodard, Classical Quantum Gravity 24, 201 (2007).

[21] T. Prokopec, N. C. Tsamis, and R. P. Woodard, Phys. Rev. D 78, 043523 (2008).

[22] T. Prokopec, N. Tsamis, and R. Woodard, Ann. Phys. (Amsterdam) 323, 1324 (2008).

[23] S.-P. Miao and R. Woodard, Phys. Rev. D 74, 044019 (2006).

[24] G. Lazzari and T. Prokopec, arXiv:1304.0404.

[25] J. Serreau, Phys. Lett. B 730, 271 (2014).

[26] T. Prokopec, J. Cosmol. Astropart. Phys. 12 (2012) 023.

[27] J. Serreau, Phys. Rev. Lett. 107, 191103 (2011).

[28] T. Janssen, S. Miao, T. Prokopec, and R. Woodard, J. Cosmol. Astropart. Phys. 05 (2009) 003. 
[29] A. A. Starobinsky and J. Yokoyama, Phys. Rev. D 50, 6357 (1994).

[30] A. Vilenkin and L. Ford, Phys. Rev. D 26, 1231 (1982).

[31] L. Ford and L. Parker, Phys. Rev. D 16, 245 (1977).

[32] N. Tsamis and R. Woodard, Classical Quantum Gravity 20, 5205 (2003).

[33] P. Ade et al. (Planck Collaboration), Astron. Astrophys. 571, A1 (2014).

[34] E. Kahya, V. Onemli, and R. Woodard, Phys. Rev. D 81, 023508 (2010).

[35] N. Tsamis and R. Woodard, Ann. Phys. (N.Y.) 253, 1 (1997).

[36] N. Tsamis and R. Woodard, Nucl. Phys. B474, 235 (1996).

[37] W. Suen and P. Anderson, Phys. Rev. D 35, 2940 (1987).

[38] T. S. Koivisto and T. Prokopec, Phys. Rev. D 83, 044015 (2011).
[39] T. Janssen and T. Prokopec, Classical Quantum Gravity 25, 055007 (2008).

[40] J. F. Koksma and T. Prokopec, Phys. Rev. D 78, 023508 (2008).

[41] T. Prokopec, O. Tornkvist, and R. P. Woodard, Phys. Rev. Lett. 89, 101301 (2002).

[42] T. Prokopec, O. Tornkvist, and R. Woodard, Ann. Phys. (Amsterdam) 303, 251 (2003).

[43] N. D. Birrell and P. C. W Davies, Quantum Fields in Curved Space (Cambridge University Press, Cambridge, 1982).

[44] S. Weinberg, Phys. Rev. D 83, 063508 (2011).

[45] F. Albareti, J. Cembranos, and A. Maroto, Phys. Rev. D 90, 123509 (2015).

[46] H. Sheikhahmadi, A. Aghamohammadi, and K. Saaidi, arXiv:1407.0125.

[47] D. Capper and M. Duff, Nuovo Cimento A 23, 173 (1974).

[48] M. Duff, Nucl. Phys. B125, 334 (1977). 